\title{
POSITIVE QUADRATIC DIFFERENTIAL FORMS AND FOLIATIONS WITH SINGULARITIES ON SURFACES
}

\author{
VÍCTOR GUÍÑ̃EZ
}

\begin{abstract}
To every positive $C^{r}$-quadratic differential form defined on an oriented two manifold is associated a pair of transversal one-dimensional $C^{r}$ foliations with common singularities. An open set of positive $C^{r}$-quadratic differential forms with structural stable associated foliations is characterized and it is proved that this set is dense in the space of positive $C^{\infty}$-quadratic differential forms with $C^{2}$-topology. Also a realization theorem is established.
\end{abstract}

1. Introduction. A positive $C^{r}$-quadratic differential form on an oriented two-dimensional manifold (see $\S 2$ for definitions) has associated two transverse $C^{r}$ one-dimensional foliations with common singularities called the configuration of the quadratic differential form. The basic problems to be considered here are the local and global descriptions of these configurations, their (structural) stability under small perturbations of the positive quadratic differential form, and the genericity of the stability property.

Another question that we will consider is the realization by a positive $C^{r}$ quadratic differential form of an arbitrary configuration of two transverse $C^{r}$-onedimensional foliations with common singularities.

In 1952 Hartman and Wintner [3] studied the existence of spiral solutions in the neighborhood of a singularity for continuous positive quadratic differential forms and applied their results to lines of principal curvature and to asymptotic lines around umbilical points.

Later in 1982, Sotomayor and Gutiérrez $[\mathbf{2}, \mathbf{1 2}]$ considered immersions in $\mathbf{R}^{3}$ of a compact oriented two manifold. They described a class of immersions with stable configuration of lines of principal curvature and they proved that this class is dense in the space of immersions with the $C^{2}$-topology. We recall that the configuration of lines of principal curvature is obtained as the configuration of a positive quadratic differential form.

In our work we consider the space of positive $C^{r}$-quadratic differential forms on an oriented compact two manifold. We describe a class of such forms each of whose elements has stable configuration and we prove that this class is dense in the space of positive $C^{\infty}$-quadratic differential forms with the $C^{2}$-topology.

Received by the editors November 10, 1986 .

1980 Mathematics Subject Classification (1985 Revision). Primary 57R30; Secondary 58F10, $34 \mathrm{C} 04$.

The author acknowledges the very kind hospitality provided by IMPA/CNP ${ }_{q}$ during the preparation of this paper. This work was partially supported by $\mathrm{CNP}_{q}$ (Brasil) and PNUD-UNESCO CHI-84-004. 
We also prove in this paper (Realization Theorem) that every configuration of two transverse $C^{r}$-one-dimensional foliations with common singularities is realized as the configuration of a positive $C^{r}$-quadratic differential form.

This paper is organized as follows. $§ 2$ contains the principal definitions and the precise statement of the results, namely Theorem A and Theorem B. In $\S 3$ we define the set $S_{r}(M)$ (Theorem B) whose elements are proved to be structural stable positive $C^{r}$-quadratic differential forms. The proof of Theorem A (Realization Theorem) is presented in $\S 4$. The next sections are devoted to the proof of Theorem B. In $\S 5$ we prove the openness of $G_{r}(M)$, the set of positive $C^{r}$-quadratic differential forms with simple singular points. In $\S 6$ we prove the openness of the set $S_{r}(M)$ and the structural stability of its elements. In $\S 7$ we prove the density of the set $S_{r}(M)$ in $G_{r}(M)$ with the $C^{s}$-topology for any $s \leq r$. Finally in the last sections we complete the proof of Theorem B proving the density of the set $G_{\infty}(M)$ in the set of positive $C^{\infty}$-quadratic differential forms with with $C^{2}$-topology.

This work corresponds to my doctoral thesis at IMPA. I wish to thank my adviser C. Gutiérrez who suggested this problem. Thanks are also due to J. Palis and C. Gutiérrez for their help and interest in this work.

2. Definitions and statement of results. This section contains our principal definitions and the precise statement of our results. By $M$ we will denote a $C^{\infty}$ compact, connected, oriented two-dimensional manifold.

2.1. Definition. A $C^{r}$-quadratic differential form on $M$ is an element of the form $w=\sum_{i=1}^{n} \phi_{i} \psi_{i}$, where $\phi_{i}$ and $\psi_{i}$ are $C^{r} 1$-forms on $M$. In other words, for each point $p$ in $M, w(p)=\sum_{i=1}^{n} \phi_{i}(p) \psi_{i}(p): T_{p} M \rightarrow \mathbf{R}$ is the map defined by $w(p)(v)=\sum_{i=1}^{n} \phi_{i}(p)(v) \cdot \psi_{i}(p)(v)$ for all vectors $v$ in $T_{p} M$.

If $f: M \rightarrow N$ is a $C^{r+1}$-diffeomorphism and $w$ is a $C^{r}$-quadratic differential form on $M$, we denote by $f^{*}(w)$ the $C^{r}$-quadratic differential form on $N$, defined by $f^{*}(w)(q)(v)=w\left(f^{-1}(q)\right)\left(d f_{q}^{-1}(v)\right)$ for $q$ in $N$ and $v$ in $T_{q} N$.

Observe that if $(x, y): U \subseteq M \rightarrow \mathbf{R}^{2}$ is a chart and $w$ is a $C^{r}$-quadratic differential form then $(x, y)^{*}(w)$ is of the form: $a(x, y) d y^{2}+b(x, y) d x d y+c(x, y) d x^{2}$, where $a, b, c$ are real-valued functions of class $C^{r}$ defined in $(x, y)(U)$ and $d x$ and $d y$ are the projections $d x(x, y)=x$ and $d y(x, y)=y$. Moreover, if $g=\left(g_{1}, g_{2}\right): \mathbf{R}^{2} \rightarrow \mathbf{R}^{2}$ is a $C^{r+1}$ diffeomorphism and $(u, v)=g^{-1} \circ(x, y)$ then $(u, v)^{*}(w)=\tilde{a}(u, v) d v^{2}+$ $\tilde{b}(u, v) d u d v+\tilde{c}(u, v) d u^{2}$, where

$$
\begin{aligned}
\tilde{a}= & (a \circ g)\left(\frac{\partial g_{2}}{\partial v}\right)^{2}+(b \circ g)\left(\frac{\partial g_{1}}{\partial v} \cdot \frac{\partial g_{2}}{\partial v}\right)+(c \circ g)\left(\frac{\partial g_{1}}{\partial v}\right)^{2}, \\
\tilde{b}= & 2(a \circ g)\left(\frac{\partial g_{2}}{\partial u} \cdot \frac{\partial g_{2}}{\partial v}\right)+(b \circ g)\left(\frac{\partial g_{1}}{\partial u} \cdot \frac{\partial g_{2}}{\partial v}+\frac{\partial g_{1}}{\partial v} \cdot \frac{\partial g_{2}}{\partial u}\right) \\
& +2(c \circ g)\left(\frac{\partial g_{1}}{\partial u} \cdot \frac{\partial g_{1}}{\partial v}\right), \\
\tilde{c}= & (a \circ g)\left(\frac{\partial g_{2}}{\partial u}\right)^{2}+(b \circ g)\left(\frac{\partial g_{1}}{\partial u} \cdot \frac{\partial g_{2}}{\partial u}\right)+(c \circ g)\left(\frac{\partial g_{1}}{\partial u}\right)^{2} .
\end{aligned}
$$

2.2. Definition. A positive $C^{r}$-quadratic differential form on $M$ is a $C^{r}$ quadratic differential form $w$ such that for every point $p$ in $M$ the subset $w(p)^{-1}(0)$ 
sf $T_{p} M$ is either

(i) the union of two transversal lines (in which case $p$ is called a regular point of $w)$, or

(ii) all $T_{p} M$ (in which case $p$ is called a singular point of $w$ ).

The set of all positive $C^{r}$-quadratic differential forms on $M$ will be denoted by $\mathscr{F}_{r}(M)$ and when endowed with the $C^{s}$-Whitney topology we will denote it by $\mathscr{F}_{r} s(M)$.

2.3. REMARK. Let $w$ be a $C^{r}$-quadratic differential form on $M$. Then the following properties are equivalent:

(a) $w$ is in $\mathscr{F}_{r}(M)$.

(b) For each point $p$ in $M$, there exists a local chart in $p,(x, y): U \rightarrow \mathbf{R}^{2}$ such that if $(x, y)^{*}(w)=a(x, y) d y^{2}+b(x, y) d x d y+c(x, y) d x^{2}$ then $b^{2}-4 a c \geq 0$ on $(x, y)(U)$ and $\left(b^{2}-4 a c\right)^{-1}(0)=a^{-1}(0) \cap b^{-1}(0) \cap c^{-1}(0)$.

(c) For each point $p$ in $M$, the statement (b) holds for every local chart in $p$.

2.4. Definition. A $C^{r}$-configuration on $M$ is a triple $\left\{f_{1}, f_{2}, S\right\}$ where $S$ is a closed subset of $M$ and $f_{1}, f_{2}$ are two transverse $C^{r}$-one-dimensional foliations on $M-S$.

Observe that each $w$ in $\mathscr{F}_{r}(M)$ defines a configuration (called the configuration of $w) C(w)=\left\{f_{1}(w), f_{2}(w)\right.$, $\left.\operatorname{Sing}(w)\right\}$, where $\operatorname{Sing}(w)$ is the set of singular points of $w$ and $f_{1}(w), f_{2}(w)$ are the transverse foliations on $M-\operatorname{Sing}(w)$ whose tangent lines at each regular point $p$ are given by the transverse lines of $w(p)^{-1}(0)$.

2.5. REMARK. Let $w$ be in $\mathscr{F}_{r}(M)$ and let $(x, y): U \rightarrow \mathbf{R}^{2}$ be a chart. If $(x, y)^{*}(w)=a(x, y) d y^{2}+b(x, y) d x d y+c(x, y) d x^{2}$ then the foliation $f_{1}\left((x, y)^{*}(w)\right)$ (respectively $f_{2}\left((x, y)^{*}(w)\right)$ ) is tangent to the vector field

$$
\dot{x}=2 a(x, y), \quad \dot{y}=-b(x, y)+(-1)^{j} \sqrt{\left(b^{2}-4 a c\right)(x, y)}
$$

for $j=0$ (resp. $j=1$ ) or $j=1$ (resp. $j=0$ ) except possibly when $a=0$.

2.6. DEFINITION. Two forms $w_{1}$ and $w_{2}$ in $\mathscr{F}_{r}(M)$ are said to be equivalent if there exists a homeomorphism $h: M \rightarrow M$ such that $h\left(C\left(w_{1}\right)\right)=C\left(w_{2}\right)$. That is, $h\left(\operatorname{Sing}\left(w_{1}\right)\right)=\operatorname{Sing}\left(w_{2}\right)$ and $h / M-\operatorname{Sing}\left(w_{1}\right)$ maps the foliations of $w_{1}$ onto the foliations of $w_{2}$.

Observe that if $w_{1}$ and $w_{2}$ are in $\mathscr{F}_{r}(M)$ and if $h: M \rightarrow M$ is an equivalence between $w_{1}$ and $w_{2}$, then $C\left(w_{2}\right)=\left\{h\left(f_{1}\left(w_{1}\right)\right), h\left(f_{2}\left(2_{1}\right)\right), h\left(\operatorname{Sing}\left(w_{1}\right)\right)\right\}$.

2.7. Definition. A positive $C^{r}$-quadratic differential form $w_{0}$ on $M$ is said to be structurally stable if there exists a neighborhood $\mathscr{N}\left(w_{0}\right)$ of $w_{0}$ in $\mathscr{F}_{r}^{1}(M)$ such that every $w$ in $\mathscr{N}\left(w_{0}\right)$ is equivalent to $w_{0}$.

To give the next definition, notice that every $C^{r}$-quadratic differential form $w$ on $M$ can be considered as a $C^{r}$-map, which we denote by the same letter $w$, from $M$ to the $C^{\infty}$-manifold of dimension five $Q(M)=\left\{(p, \alpha) \mid p \in M\right.$ and $\alpha=\sum_{i=1}^{n} \phi_{i} \psi_{i}$ with $\phi_{i}$ and $\psi_{i}$ in the cotangent space $\left.\left(T_{p} M\right)^{*}\right\}$, in such a way that $\pi_{1} w=1_{M}$, where $\pi_{1}$ is the projection $\pi_{1}(p, \alpha)=p$. Observe that if $\left\{U_{i}, \phi_{i}\right\}$ is an atlas over $M$, then $\left\{Q\left(U_{i}\right) \bar{\phi}_{i}\right\}$ is an atlas over $Q(M)$, where $Q\left(U_{i}\right)=\left\{(p, \alpha) \in Q(M) \mid p \in U_{i}\right\}$ and $\bar{\phi}_{i}: Q\left(U_{i}\right) \rightarrow \mathbf{R}^{5}$ is defined by $\bar{\phi}_{i}(p, \alpha)=(x(p), y(p), a(p), b(p), c(p))$, where $\phi_{i}=(x, y)$ and $\alpha=a(p) d y^{2}+b(p) d x d y+c(p) d x^{2}$. Here $d x, d y: T_{p} M \rightarrow \mathbf{R}$ are the projections associated to the basis of $T_{p} M$ induced by the chart $(x, y)$. With this notation, the usual derivate of $w$ at each $p$ in $M, D w_{p}$, is a quadratic differential form on $T_{p} M$. 
2.8. Definition. A singular point $p$ of $w$ in $\mathscr{F}_{r}(M)$ is said to be a simple singular point of $w$ if $D w_{p}$ is a positive quadratic differential form on $T_{p} M$.

2.9. REMARK. We will see later that in an appropriate local chart $(x, y):(U, p)$ $\rightarrow\left(\mathbf{R}^{2}, 0\right)$ every simple singularity $p$ of $w$ in $\mathscr{F}_{r}(M)$ can be expressed in the form:

$$
\begin{aligned}
(x, y)^{*}(w)= & \left(y+M_{1}(x, y)\right) d y^{2}+\left(b_{1} x+b_{2} y+M_{2}(x, y)\right) d x d y \\
& +\left(-y+M_{3}(x, y)\right) d x^{2} \quad \text { with } M_{i}(x, y)=O\left(x^{2}+y^{2}\right),
\end{aligned}
$$

$i=1,2,3$ and $b_{1} \neq 0$.

The set of $w$ in $\mathscr{F}_{r}(M)$ such that all its singular points are simple will be denoted by $G_{r}(M)$.

Our principal results are the following two theorems:

2.10. THEOREM A. (a) Given a $C^{r}$-configuration $\left\{f_{1}, f_{2}, S\right\}$ on $M$, there exists $w$ in $\mathscr{F}_{r}(M)$ for which $C(w)=\left\{f_{1}, f_{2}, S\right\}$.

(b) If $w_{1}$ and $w_{2}$ in $\mathscr{F}_{r}(M)$ are such that $C\left(w_{1}\right)=C\left(w_{2}\right)$, then $w_{1}=\lambda w_{2}$ where $\lambda$ is a $C^{r}$-function $\lambda: M-\operatorname{Sing}\left(w_{1}\right) \rightarrow \mathbf{R}-\{0\}$.

2.11. COROLLARY. Given a $C^{r}$-one-dimensional foliation $f$ on $M-S$, where $S$ is a closed subset of $M$, there exists $w \in \mathscr{F}_{r}(M)$ such that $S=\operatorname{Sing}(w)$ and $f=f_{1}(w)$.

2.12. REMARK. The configuration of lines of principal curvature of an immersion of $M$ in $\mathbf{R}^{3}[\mathbf{2}, \mathbf{1 2}]$; the configuration of a pseudo-Anosov diffeomorphism [10]; the measured foliations $[\mathbf{4}, \mathbf{1 0}]$; the arational foliations $[\mathbf{9}]$, the line fields $[\mathbf{1}, \mathbf{5}]$, and the flow of vector fields are all realized as configurations of positive quadratic differential forms.

2.13. THEOREM B. For each $1 \leq r \leq \infty$, there exists a nonempty open subset $S_{r}(M) \subseteq G_{r}(M)$ of $\mathscr{F}_{r}^{1}(M)$ such that:

(i) All elements in $S_{r}(M)$ are structurally stable.

(ii) $S_{r}(M)$ is dense in $G_{r}(M)$ with the $C^{s}$-topology for $s \leq r$.

(iii) $G_{\infty}(M)$ is dense in $\mathscr{F}_{\infty}^{2}(M)$ and therefore $S_{\infty}(M)$ is dense in $\mathscr{F}_{\infty}^{2}(M)$.

2.14. REMARKs. (i) From Theorem B it follows that the structurally stable smooth positive quadratic differential forms are $C^{2}$-generic.

(ii) In the proof of Theorem B we used the Weierstrass $C^{\infty}$-Preparation Theorem and the Mather $C^{\infty}$-Division Theorem: therefore our proof cannot be extended for the $C^{r}$-case.

3. The set $S_{r}(M)$. In this section we introduce the set $S_{r}(M)$ that appears in the statement of Theorem B above. The elements of $S_{r}(M)$ will be defined in terms of the singular points, the compact leaves, and the asymptotic behavior of noncompact leaves, especially singular separatrices. The precise definition of these concepts is given below.

3.1. DEFINITION. Let $p$ be a simple singular point of $w$ in $\mathscr{F}_{r}(M)$. We say that $p$ is a hyperbolic singular point of $w$ if the homogeneous polynomial of degree $3,\left(D w_{p}\right)(x, y)(x, y)$, has only simple roots.

To obtain the local configuration of $w$ in a neighborhood of a hyperbolic singular point $p$, consider a local chart $(x, y):(U, p) \rightarrow\left(\mathbf{R}^{2}, 0\right)$ such that

$$
\begin{aligned}
(x, y)^{*}(w)= & \left(y+M_{1}(x, y)\right) d y^{2}+\left(b_{1} x+b_{2} y+M_{2}(x, y)\right) d x d y \\
& +\left(-y+M_{3}(x, y)\right) d x^{2}
\end{aligned}
$$


with $M_{i}(x, y)=O\left(x^{2}+y^{2}\right), i=1,2,3$ and $b_{1} \neq 0$ (Remark 2.9).

Then $D(x, y)_{p}^{*}\left(D w_{p}\right)=(u, v)^{*}\left(D w_{p}\right)=v d v^{2}+\left(b_{1} u+b_{2} v\right) d u d v-v d u^{2}$ and $(u, v)^{*}\left(D w_{p}\right)\left(v_{1}, v_{2}\right)\left(v_{1}, v_{2}\right)=v_{2}\left[v_{2}^{2}+b_{2} v_{1} v_{2}+\left(b_{1}-1\right) v_{1}^{2}\right]$.

The conditions for hyperbolicity are given by:

$$
\left(b_{2} / 2\right)^{2}+1-b_{1} \neq 0 \text { and } \quad b_{1} \neq 1 \text {. }
$$

Since $b_{1}$ does not vanish, we have the following three cases:

$$
\begin{aligned}
& \mathrm{D}_{1}:\left(b_{2} / 2\right)^{2}+1<b_{1}, \\
& \mathrm{D}_{2}:\left(b_{2} / 2\right)^{2}+1>b_{1}>0 \text { and } b_{1} \neq 1, \\
& \mathrm{D}_{3}: b_{1}<0 .
\end{aligned}
$$

As we will see the corresponding local configurations of $w$ at $p$ are the ones given in Figure 1.

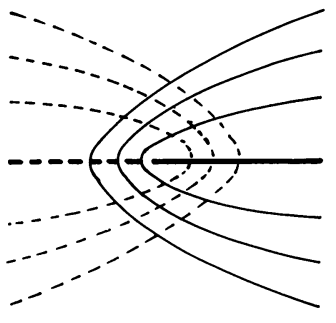

Case $D_{1}$

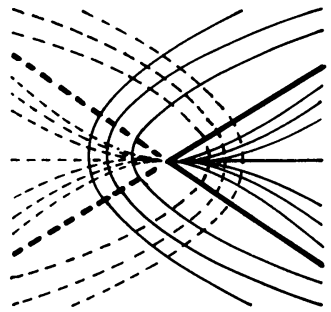

Case $\mathrm{D}_{2}$

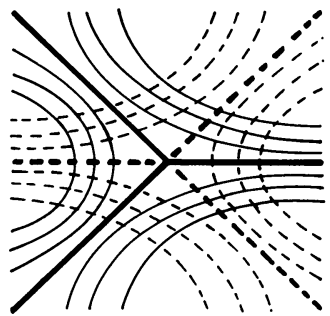

Case $\mathrm{D}_{3}$

FIGURE 1

3.2. REMARKS. 1. The foliations $f_{1}(w)$ and $f_{2}(w)$ above are unoriented on a neighborhood of the singular point. The Poincaré index in the cases $D_{1}$ and $D_{2}$ is $1 / 2$ and in the case $D_{3}$ is $-1 / 2$.

2. In Figure 1, the separatrices are drawn in heavy lines. These are leaves which approach the singular points and which separate regions of different patterns of approach to these points. Therefore, in case $\mathrm{D}_{i}, i=1,2$ or 3 , we have $i$-separatrices.

3.3. DEFINITION. Let $w$ be in $\mathscr{F}_{r}(M)$ and let $c$ be a compact leaf of $w$. We say that $c$ is a hyperbolic compact leaf of $w$ if the Poincaré first return map $P$ associated to a transversal line to $c$ at a point $q \in c$ verifies $P^{\prime}(q) \neq 1$.

3.4. Definition. Let $S_{r}(M)$ be the set of $w$ in $G_{r}(M)$ such that:

(a) $w$ has a finite number of singular points and compact leaves, all of which are hyperbolic.

(b) The limit set of every leaf of $f_{i}(w), i=1,2$, is the union of singular points and compact leaves.

(c) $w$ has no double separatrices. That is, there is no separatrix of a singular point of $w$ which is a separatrix of two different singular points or twice a separatrix of the same singular point.

\section{Proof of Theorem A (Realization Theorem).}

4.1. THEOREM A (PART (a)). Take a smooth riemannian metric on $M$.

Let $C$ be a connected component of $M-S$. Fix $p \in C$ and consider two unit vectors $v_{1}$ and $v_{2}$ of $T_{p} M$ tangent at $p$ to $f_{1}$ and $f_{2}$ respectively. Define $\tilde{w}(p)=\theta_{1}(p) \cdot \theta_{2}(p)$, where $\theta_{i}(p) \in\left(T_{p} M\right)^{*}, i=1,2$, satisfies $\theta_{i}(p)\left(v_{j}\right)=1-\delta_{i j}$, $j=1,2$. 
Let $q$ be an arbitrary point of $C$. In order to define $\tilde{w}(q)$ we consider a regular curve $l \subset C$ without self-intersections connecting $p$ and $q$. Along $l$ we may define differentiable unit vector fields $V_{i}, i=1,2$, and 1-forms $\theta_{j}, j=1,2$, uniquely determined by the conditions that $V_{i}(p)=v_{i}, V_{i}$ is tangent to $f_{i}$, and $\theta_{j}\left(V_{i}\right)=$ $1-\delta_{i j}$. Under these circumstances we define $\tilde{w}(q)=\theta_{1}(q) \cdot \theta_{2}(q)$. Using the fact that $M$ is orientable and that $f_{1}$ and $f_{2}$ are pairwise transversal, we can easily see that this definition does not depend on the particular curve $l$ connecting $p$ and $q$. Therefore, since $f_{1}$ and $f_{2}$ are of class $C^{r}$ we have that $\tilde{w}$ is defined in $M-S$ and is of class $C^{r}$ too.

If $S=\varnothing$ the theorem is proved. So assume $S \neq \varnothing$. Then we may find a sequence $\left\{U_{i}\right\}$ of open subsets of $M$ such that $U_{1} \supset \bar{U}_{2} \supset U_{2} \supset \bar{U}_{3} \supset \cdots \supset S$ and $\bigcap_{i=1}^{\infty} U_{i}=S$. Let $A_{0}=M-\bar{U}_{2}$ and $A_{i}=U_{i}-\bar{U}_{i+3}$ for $i \geq 1$. Then $\left\{A_{i}\right\}_{i=0}^{\infty}$ is a locally finite open covering of $M-S$. Let $\sum_{i=0}^{\infty} \phi_{i}$ be a smooth partition of unity strictly subordinate to $\left\{A_{i}\right\}_{i=0}^{\infty}$. Take a sequence $\left\{c_{i}\right\}_{i=0}^{\infty}$ of positive real numbers such that for all $i \in \mathbf{N} c_{i} \cdot\left\|\phi_{i} \cdot \tilde{w}\right\|_{i}<1 / 2^{i}$ on $A_{i}$ and define

$$
w= \begin{cases}\left(\sum_{i=0}^{\infty} c_{i} \cdot \phi_{i}\right) \cdot \tilde{w} & \text { in } M-S, \\ 0 & \text { in } S .\end{cases}
$$

Therefore, $w \in \mathscr{F}_{r}(M)$ and $C(w)=\left\{f_{1}, f_{2}, S\right\}$. This finishes the proof of Theorem $\mathrm{A}(\mathrm{a})$.

The second part of Theorem A will be a consequence of the following lemma.

4.2. Lemma. Let $w, \tilde{w} \in \mathscr{F}_{r}(M)$ and $V \subseteq M$ be an open subset contained in $(M-\operatorname{Sing}(w)) \cap(M-\operatorname{Sing}(\tilde{w}))$. If $w / V$ and $\tilde{w} / V$ have the same associated foliations, there exists a $C^{r}$-function $\lambda: V \rightarrow \mathbf{R}-\{0\}$ such that $\tilde{w}(p)=\lambda(p) w(p)$ for all $p \in V$.

Proof. Let $(x, y): U \subseteq V \rightarrow \mathbf{R}^{2}$ be a chart. Let the local expression of $w$ and $\tilde{w}$ in this chart be

$$
\begin{aligned}
& (x, y)^{*}(w)=a(x, y) d y^{2}+b(x, y) d x d y+(x, y) d x^{2} \\
& (x, y)^{*}(\tilde{w})=\tilde{a}(x, y) d y^{2}+\tilde{b}(x, y) d x d y+\tilde{c}(x, y) d x^{2} .
\end{aligned}
$$

The fact that $(x, y)^{*}(w)$ and $(x, y)^{*}(\tilde{w})$ determine the same foliations in $(x, y)(U)$ is equivalent to the fact that for any $\left(x_{0}, y_{0}\right)$ in $(x, y)(U)$, the real quadratic polynomials in the $z$-variable

$$
a\left(x_{0}, y_{0}\right) z^{2}+b\left(x_{0}, y_{0}\right) z+c\left(x_{0}, y_{0}\right) \quad \text { and } \quad \tilde{a}\left(x_{0}, y_{0}\right) z^{2}+\tilde{b}\left(x_{0}, y_{0}\right) z+\tilde{c}\left(x_{0}, y_{0}\right)
$$

have the same roots. Therefore there exists a function $\lambda: U \rightarrow \mathbf{R}-\{0\}$ such that

$$
(\tilde{a}, \tilde{b}, \tilde{c})(x, y)(p)=\lambda(p) \cdot(a, b, c)(x, y)(p) .
$$

Certainly, $\lambda$ is of class $C^{r}$ and does not depend on the particular chart. This finishes the proof.

5. Simple singular points. This section contains the proof of the openness of the set $G_{r}(M)$ (defined in $\S 2$ ) in $\mathscr{F}_{r}^{1}(M)$. The following propositions characterize the simple singular points. 
5.1. Proposition. Let $p$ be a singular point of $w$ in $\mathscr{F}_{r}(M)$. The following statements are equivalent:

(1) The point $p$ is a simple singular point of $w$.

(2) There exists a local chart $(x, y):(U, p) \rightarrow\left(\mathbf{R}^{2}, 0\right)$ such that if $(x, y)^{*}(w)=$ $\left(a_{1} x+a_{2} y+M_{1}(x, y)\right) d y^{2}+\left(b_{1} x+b_{2} y+M_{2}(x, y)\right) d x d y+\left(c_{1} x+c_{2} y+M_{3}(x, y)\right) d x^{2}$ with $M_{i}(x, y)=O\left(x^{2}+y^{2}\right), i=1,2,3$, then

$$
b_{1}^{2}-4 a_{1} c_{1}>0
$$

and

$$
\left(b_{1}^{2}-4 a_{1} c_{1}\right)\left(b_{2}^{2}-4 a_{2} c_{2}\right)-\left(b_{1} b_{2}-2 a_{1} c_{2}-2 a_{2} c_{1}\right)^{2}>0 .
$$

(3) The statement (2) holds for every local chart in $p$.

(4) There exists a local chart $(x, y):(U, p) \rightarrow\left(\mathbf{R}^{2}, 0\right)$ such that

$$
\begin{aligned}
(x, y)^{*}(w)= & \left(y+M_{1}(x, y)\right) d y^{2}+\left(b_{1} x+b_{2} y+M_{2}(x, y)\right) d x d y \\
& +\left(-y+M_{3}(x, y)\right) d x^{2}
\end{aligned}
$$

with $M_{i}(x, y)=O\left(x^{2}+y^{2}\right), i=1,2,3$ and $b_{1} \neq 0$.

PROOF. The equivalence between (1), (2), and (3) is a consequence of the following facts:

(a) If $\left\{U_{1}, \phi_{i}\right\}$ is an atlas over $M$, then $\left\{Q\left(U_{i}\right), \bar{\phi}_{i}\right\}$ is an atlas over $Q(M)$, where $Q\left(U_{i}\right)=\left\{(p, \alpha) \in Q(M) \mid p \in U_{i}\right\}$ and $\bar{\phi}_{i}: Q\left(U_{i}\right) \rightarrow \mathbf{R}^{5}$ is defined by $\bar{\phi}_{i}(p, \alpha)=$ $(x(p), y(p), a(p), b(p), c(p))$, where $\phi_{i}=(x, y)$ and $\alpha=a(p) d y^{2}+b(p) d x d y+c(p) d x^{2}$.

(b) If $(x, y):(U, p) \rightarrow\left(\mathbf{R}^{2}, 0\right)$ is a local chart and

$$
\begin{aligned}
(x, y)^{*}(w)= & \left(a_{1} x+a_{2} y+M_{1}(x, y)\right) d y^{2}+\left(b_{1} x+b_{2} y+M_{2}(x, y)\right) d x d y \\
& +\left(c_{1} x+c_{2} y+M_{3}(x, y)\right) d x^{2}
\end{aligned}
$$

with $M_{i}(x, y)=O\left(x^{2}+y^{2}\right)$, then $(u, v)^{*}\left(D w_{p}\right)=\left(a_{1} u+a_{2} v\right) d v^{2}+\left(b_{1} u+b_{2} v\right) d u d v+$ $\left(c_{1} u+c_{2} v\right) d u^{2}$, where $(u, v)=D(x, y)_{p}: T_{p} M \rightarrow \mathbf{R}^{2}$.

Therefore only $(2) \Rightarrow(4)$ needs to be proved. For this, let $(x, y):(U, p) \rightarrow\left(\mathbf{R}^{2}, 0\right)$ be a chart such that

$$
\begin{aligned}
(x, y)^{*}(w)= & \left(a_{1} x+a_{2} y+M_{1}(x, y)\right) d y^{2}+\left(b_{1} x+b_{2} y+M_{2}(x, y)\right) d x d y \\
& +\left(c_{1} x+c_{2} y+M_{3}(x, y)\right) d x^{2}
\end{aligned}
$$

with $M_{i}(x, y)=O\left(x^{2}+y^{2}\right), i=1,2,3$. Suppose $b_{1}^{2}-4 a_{1} c_{1}>0$, and

$$
\left(b_{1}^{2}-4 a_{1} c_{1}\right)\left(b_{2}^{2}-4 a_{2} c_{2}\right)-\left(b_{1} b_{2}-2 a_{1} c_{2}-2 a_{2} c_{1}\right)^{2}>0 .
$$

Observe that if $A: \mathbf{R}^{2} \rightarrow \mathbf{R}^{2}$ is a linear isomorphism $(u, v)=A \circ(x, y)$, $A^{-1}(u, v)=(\alpha u+\beta v, \gamma u+\delta v)$ and $(u, v)^{*}(w)=\tilde{a}(u, v) d v^{2}+\tilde{b}(u, v) d u d v+\tilde{c}(u, v) d u^{2}$, then

$$
\left.(\tilde{a}, \tilde{b}, \tilde{c})(u, v)=\mid \begin{array}{ccc}
\delta^{2} & \beta \delta & \beta^{2} \\
2 \gamma \delta & \alpha \delta+\beta \gamma & 2 \alpha \beta \\
\gamma^{2} & \alpha \gamma & \alpha^{2}
\end{array}\right]\left|\begin{array}{l}
a\left(A^{-1}(u, v)\right) \\
b\left(A^{-1}(u, v)\right) \\
c\left(A^{-1}(u, v)\right)
\end{array}\right|
$$


and

$$
\left\{\begin{array}{l}
\tilde{a}_{1}=\frac{\partial \tilde{a}}{\partial u}(0,0)=\delta^{2}\left(\alpha a_{1}+\gamma a_{2}\right)+\beta \delta\left(\alpha b_{1}+\gamma b_{2}\right)+\beta^{2}\left(\alpha c_{1}+\gamma c_{2}\right), \\
\tilde{a}_{2}=\frac{\partial \tilde{a}}{\partial v}(0,0)=\delta^{2}\left(\beta a_{1}+\delta a_{2}\right)+\beta \delta\left(\beta b_{1}+\delta b_{2}\right)+\beta^{2}\left(\beta c_{1}+\delta c_{2}\right), \\
\tilde{c}_{1}=\frac{\partial \tilde{c}}{\partial u}(0,0)=\gamma^{2}\left(\alpha a_{1}+\gamma a_{2}\right)+\alpha \gamma\left(\alpha b_{1}+\gamma b_{2}\right)+\alpha^{2}\left(\alpha c_{1}+\gamma c_{2}\right), \\
\tilde{c}_{2}=\frac{\partial \tilde{c}}{\partial v}(0,0)=\gamma^{2}\left(\beta a_{1}+\delta a_{2}\right)+\alpha \gamma\left(\beta b_{1}+\delta b_{2}\right)+\alpha^{2}\left(\beta c_{1}+\delta c_{2}\right) .
\end{array}\right.
$$

From this we see that if $a_{1}=c_{1}=0$, then taking $\gamma=\beta=0$ we leave this condition invariant and equations $(*)$ are $\tilde{a}_{2}=\delta^{3} a_{2}, \tilde{c}_{2}=\alpha^{2} \delta c_{2}$. Since $a_{2} \cdot c_{2}<0$, it is clear that there exist $\alpha, \delta$ such that $\tilde{a}_{2}=-\tilde{c}_{2}=1$.

Now if $c_{1}=0$ taking $\gamma=0$ we leave $c_{1}=0$ invariant and it follows from equation (*) that $\tilde{a}_{1}=\alpha \delta\left(\delta a_{1}+\beta b_{1}\right)$ with $b_{1} \neq 0$. So if $\beta=\delta a_{1} / b_{1}$ we obtain $\tilde{a}_{1}=0$.

Finally if $c_{1} \neq 0$ to obtain $\tilde{c}_{1}=0$ it is enough to make $\alpha=\lambda \gamma$ with $\lambda$ a solution of the equation $c_{1} \lambda^{3}+\left(b_{1}+c_{2}\right) \lambda^{2}+\left(a_{1}+b_{2}\right) \lambda+a_{2}=0$. Thus the proof is complete.

The openeness of the set $G_{r}(M)$ in $\mathscr{F}_{r}^{1}(M)$ will be a consequence of the following proposition:

5.2. Proposition. Let $w \in \mathscr{F}_{r}(M)$ and $p \in \operatorname{Sing}(w)$. If $p$ is a simple singular point of $w$ there exist neighborhoods $\mathcal{N}(w) \subset \mathscr{F}_{r}{ }^{1}(M)$ of $w$ and $V \subset M$ of $p$ such that every $\tilde{w} \in \mathcal{N}(w)$ has a unique singular point $p(\tilde{w})$ in $V$, which is simple.

Proof. Let $(x, y):(U, p) \rightarrow\left(\mathbf{R}^{2}, 0\right)$ be a chart such that $(x, y)^{*}(w)=a(x, y) d y^{2}$ $+b(x, y) d x d y+c(x, y) d x^{2}$ with $(a, b, c)(x, y)=\left(y, b_{1} x+b_{2} y,-y\right)+\left(M_{1}, M_{2}, M_{3}\right)(x, y)$, where $b_{1} \neq 0$ and $M_{i}(x, y)=O\left(x^{2}+y^{2}\right), i=1,2,3$.

It follows from the transversal intersection of the curves $a=0$ and $c=0$ with $b=0$ at $(0,0)$, that there exist neighborhoods $V \subset(x, y)(U)$ of $(0,0)$ and $\mathscr{N}(w) \subset$ $\mathscr{F}_{r}^{1}(M)$ of $w$ such that $a^{-1}(0) \cap b^{-1}(0) \cap c^{-1}(0) \cap V=\{(0,0)\}$ and for each $\tilde{w} \in$ $\mathscr{N}(w)$, the corresponding curves $\tilde{a}=0$ and $\tilde{c}=0$ have transversal intersection with $\tilde{b}=0$ at a single point $V$, say $(x, y)\left(p_{1}(w)\right)$ and $(x, y)\left(p_{2}(w)\right)$ respectively. Since $\tilde{b}^{2}-4 \tilde{a} \tilde{c} \geq 0$, we have $p_{1}(\tilde{w})=p_{2}(\tilde{w})=p(\tilde{w})$ and therefore $\operatorname{Sing}(\tilde{w}) \cap V=\{p(\tilde{w})\}$.

Observe that, reducing $\mathscr{N}(w)$ if necessary, we have

$$
\tilde{b}_{i}^{2}-4 \tilde{a}_{i} \tilde{c}_{i}>0, \quad i=1,2,
$$

and

$$
\left(\tilde{b}_{1}^{2}-4 \tilde{a}_{1} \tilde{c}_{1}\right)\left(\tilde{b}_{2}^{2}-4 \tilde{a}_{2} \tilde{c}_{2}\right)-\left(\tilde{b}_{1} \tilde{b}_{2}-2 \tilde{a}_{1} \tilde{c}_{2}-2 \tilde{a}_{2} \tilde{c}_{1}\right)^{2}>0
$$

where

$$
\left(\tilde{a}_{1}, \tilde{b}_{1}, \tilde{c}_{1}\right)=(\partial / \partial x)(\tilde{a}, \tilde{b}, \tilde{c})((x, y)(\tilde{p}))
$$

and

$$
\left(\tilde{a}_{2}, \tilde{b}_{2}, \tilde{c}_{2}\right)=(\partial / \partial y)(\tilde{a}, \tilde{b}, \tilde{c})((x, y)(\tilde{p})) .
$$

Therefore the proof is complete.

5.3. COROLlaRY. The subset $G_{r}(M)$ of those $w$ in $\mathscr{F}_{r}(M)$ whose singular points are all simple is open in $\mathscr{F}_{r}^{1}(M)$.

PROOF. Since $M$ is compact, the proof follows from the previous proposition. 
6. Structural stability. The openness of the set $S_{r}(M)$ (defined in $\S 3$ ) in $\mathscr{F}_{r}{ }^{1}(M)$ will be a consequence of the local stability of the hyperbolic singular points and the continuity on compact parts, under small $C^{1}$-perturbations of singular separatrices, together with the local stability of hyperbolic compact leaves.

6.1. Definition. A form $w_{0}$ in $\mathscr{F}_{r}(M)$ is said to be locally stable at a point $p$ in $M$ if there exist neighborhoods $\mathscr{N}\left(w_{0}\right)$ of $w_{0}$ in $\mathscr{F}_{r}^{1}(M)$ and $U(p)$ of $p$ in $M$ such that for each $w$ in $\mathscr{N}\left(w_{0}\right)$ there is a point $q=q(w)$ in $U(p)$ and a homeomorphism $h: V(q) \rightarrow V(p)$ between neighborhoods of $q$ and $p$ such that $h(q)=p$ and such that $h$ maps the configuration of $w / V(q)$ onto the configuration of $w_{0} / V(p)$.

6.2. Proposition. Let $p$ be a hyperbolic singular point of $w$ in $\mathscr{F}_{r}(M)$. Then $w$ is locally stable at $p$.

PROOF. Since this is similar to the case of an immersion of $M$ in $\mathbf{R}^{3}$ that verifies condition D at an umbilical point (Proposition 2.1 of [12, pp. 201-206]) only an outline of the proof is given below.

Let $p$ be a hyperbolic singular point of a $w$ in $\mathscr{F}_{r}(M)$ and $(x, y):(U, p) \rightarrow\left(\mathbf{R}^{2}, 0\right)$ be a local chart such that $(x, y)^{*}(w)=a(x, y) d y^{2}+b(x, y) d x d y+c(x, y) d x^{2}$ with $(a, b, c)(x, y)=\left(y, b_{1} x+b_{2} y,-y\right)+\left(M_{1}, M_{2}, M_{3}\right)(x, y)$, where $M_{i}(x, y)=O\left(x^{2}+y^{2}\right)$, $i=1,2,3$ and $b_{1} \neq 0$.

The vector field $Y_{i}=P \partial / \partial x+Q_{i} \partial / \partial y$, where $P(x, y)=2 a(x, y)$ and $Q_{i}(x, y)=$ $-b(x, y)+(-1)^{i+1} \sqrt{\left(b^{2}-4 a c\right)(x, y)}$ for $i=1$ or $i=2$, is tangent to one of the foliations of $w$ except possibly when $P=0$.

Making

$$
H:\left\{\begin{array}{l}
x=s \\
y=t s+y(s)
\end{array}\right.
$$

where $y=y(s)$ is the unique solution of $P(s, y(s))=0$ with $y(0)=0$ (and therefore $\left.y^{\prime}(0)=0\right)$ we obtain over $\mathbf{R}^{2}-\mathbf{R} \cdot(0,1)$ the vector fields

$$
Z_{i}=H_{*} Y_{i}=S \partial / \partial s+T_{i} \partial / \partial t
$$

Therefore

$$
S(s, t)=2 t s[1+U(s, t)] \quad \text { with } U(0, t)=0
$$

and

$$
T_{i}(s, t)=s^{-1}\left[-\left(t+y^{\prime}(s)\right) S+S_{1}+(-1)^{i+1} \sqrt{S_{1}^{2}+S S_{2}}\right]
$$

where

$$
S_{1}(s, t)=-s\left[b_{1}+b_{2} t+U_{1}(s, t)\right] \quad \text { with } U_{1}(0, t)=0
$$

and

$$
S_{2}(s, t)=-2 s\left[-t+U_{2}(s, t)\right] \quad \text { with } U_{2}(0, t)=0 .
$$

Let $\tilde{Z}_{i}$ be defined by

$$
\tilde{Z}_{i}(s, t)=r_{i}(s, t) Z_{i}=\tilde{S}_{i} \frac{\partial}{\partial s}+\tilde{T} \frac{\partial}{\partial t}
$$

where

$$
r_{i}(s, t)=(t s)^{-1}\left[-\left(t+y^{\prime}(s)\right) S+S_{1}+(-1)^{i} \sqrt{S_{1}^{2}+S S_{2}}\right] .
$$


Hence

where

$$
\tilde{S}_{i}(s, t)= \begin{cases}2 s[1+U(s, t)] f_{i}(s, t) & \text { if } s>0 \\ 2 s[1+U(s, t)] f_{3-i}(s, t) & \text { if } s<0\end{cases}
$$

$$
\begin{aligned}
f_{i}(s, t)= & 2 t\left(t+y^{\prime}(s)\right)(1+U(s, t))-\left(b_{1}+b_{2} t+U_{1}(s, t)\right) \\
& +(-1)^{i} \sqrt{\left(b_{1}+b_{2} t+U_{1}(s, t)\right)^{2}-4 t(1+U(s, t))\left(-t+U_{2}(s, t)\right)}
\end{aligned}
$$

for $i=1,2$; and

$$
\begin{aligned}
\tilde{T}(s, t)=4(1+U(s, t))\left[t\left(t+y^{\prime}(s)\right)^{2}(1+U(s, t))\right. \\
+\left(t+y^{\prime}(s)\right)\left(b_{1}+b_{2} t+U_{1}(s, t)\right)+\left(-t+U_{2}(s, t)\right] .
\end{aligned}
$$

Observe that
(a) $f_{1}(s, t) \cdot f_{2}(s, t)=t \tilde{T}(s, t)$,
(b) $\tilde{T}(0, t)=4 t\left[t^{2}+b_{2} t+b_{1}-1\right]$,
(c) $f_{1}(s, t) \leq f_{2}(s, t)$
(d) $f_{i}(0, t)=0 \Rightarrow f_{3-i}(0, t) \neq 0$.

Let us consider $i=1$.

Case $\mathrm{D}_{1} \cdot\left(\left(b_{2} / 2\right)^{2}+1-b_{1}<0\right)$.

In this case $b_{1}>0, \partial S_{1} / \partial s^{+}(0,0)=-2 b_{1}<0, \partial S_{1} / \partial s^{-}(0,0)=0, \partial \tilde{S}_{1} / \partial t(0,0)$ $=0, \partial \tilde{T} / \partial t(0,0)=4\left(b_{1}-1\right)>0$ and the phase portraits of $\tilde{Z}_{1}$ and $Y_{1}$ are as shown in Figure 2.
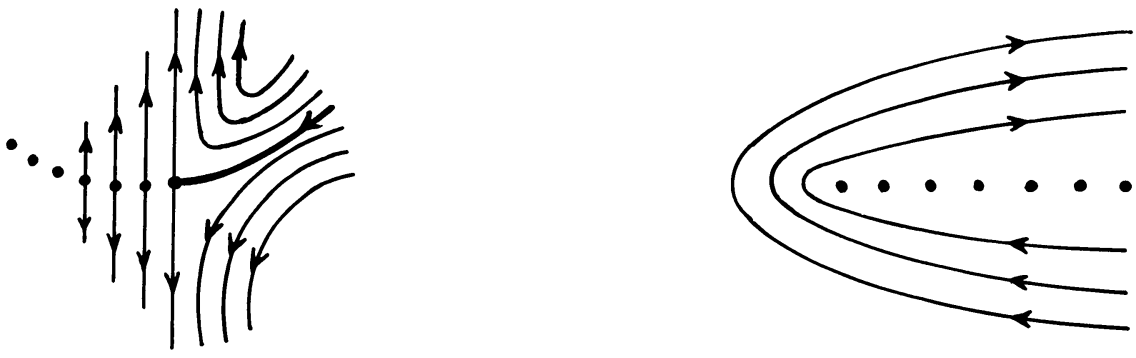

FIGURE 2

Cases $\mathrm{D}_{2}$ and $\mathrm{D}_{3}$. $\left(\left(b_{2} / 2\right)^{2}+1-b_{1}>0\right)$.

In order to simplify the analysis of these cases we suppose that $1-b_{1}>0$. Therefore $f_{1}(0, t)<0$ and $t_{2}<0<t_{1}$, where

$$
t_{1}=-\frac{b_{2}}{2}+\sqrt{\left(\frac{b_{2}}{2}\right)^{2}+1-b_{1}} \quad \text { and } \quad t_{2}=-\frac{b_{2}}{2}-\sqrt{\left(\frac{b_{2}}{2}\right)^{2}+1-b_{1}} .
$$

Hence

(a)

$$
\begin{gathered}
\frac{\partial \tilde{S}_{1}}{\partial s^{+}}(0,0)=-b_{1}-\left|b_{1}\right|, \quad \frac{\partial \tilde{S}_{1}}{\partial s^{-}}(0,0)=-b_{1}+\left|b_{1}\right| \\
\frac{\partial \tilde{S}_{1}}{\partial t}(0,0)=0 \quad \text { and } \quad \frac{\partial \tilde{T}}{\partial t}(0,0)=4\left(b_{1}-1\right)<0 .
\end{gathered}
$$


(b)

$$
\begin{gathered}
\frac{\partial \tilde{S}_{1}}{\partial s^{+}}\left(0, t_{i}\right)=2 f_{1}\left(0, t_{i}\right)<0, \quad \frac{\partial \tilde{S}_{1}}{\partial s^{-}}\left(0, t_{i}\right)=0, \quad \frac{\partial \tilde{S}_{1}}{\partial t}\left(0, t_{i}\right)=0 \\
\frac{\partial \tilde{T}}{\partial t}\left(0, t_{i}\right)=(-1)^{i+1} 8 t_{i} \sqrt{\left(\frac{b_{2}}{2}\right)^{2}+1-b_{1}}>0 \quad \text { for } i=1,2 .
\end{gathered}
$$

Therefore the phase portraits of $\tilde{Z}_{1}$ and $Y_{1}$ are as shown in Figure 3.
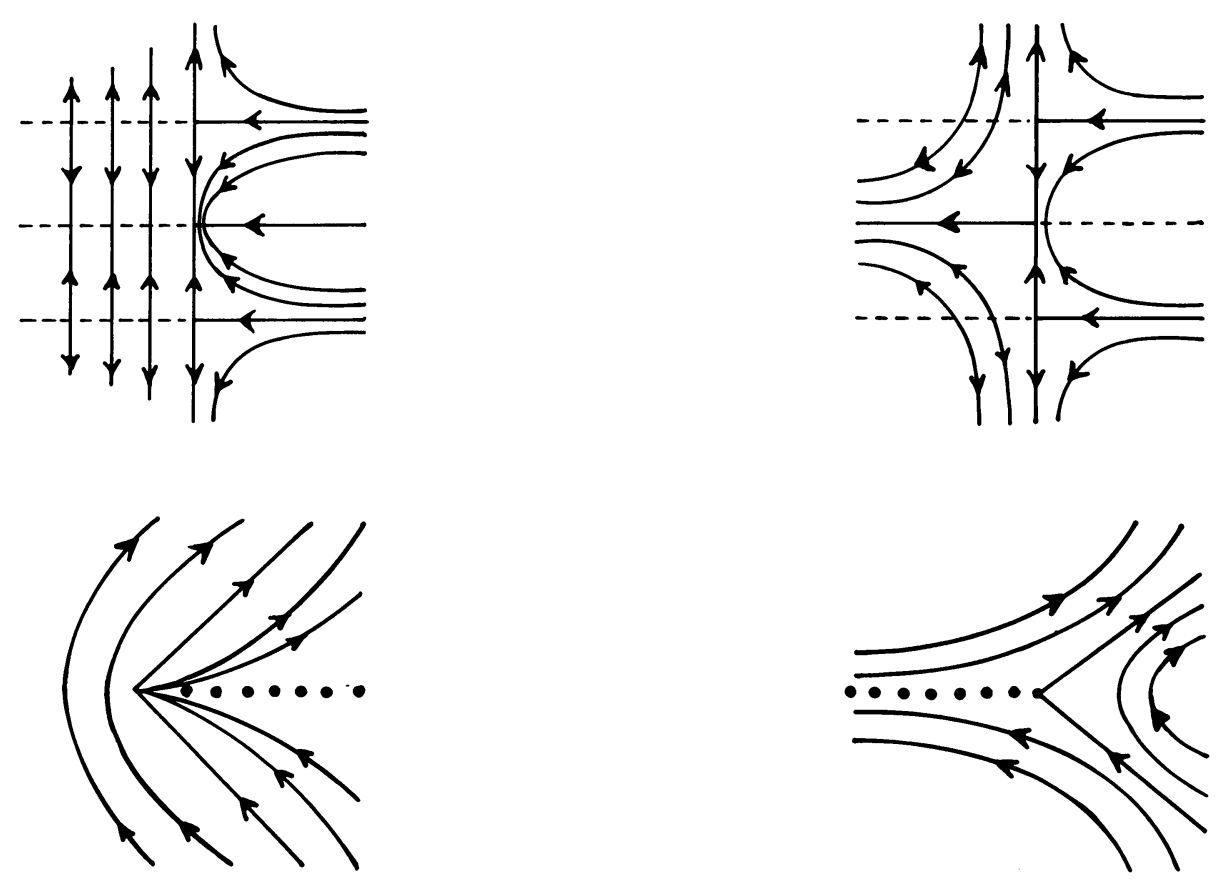

FIGURE 3

Case $\mathrm{D}_{2} \cdot\left(\left(b_{2} / 2\right)^{2}+1>b_{1}>0\right) . \quad$ Case $\mathrm{D}_{3} .\left(b_{1}<0\right)$.

The construction of the topological equivalence at each case is straightfoward.

6.3. DEFINITION. A form $w_{0}$ in $\mathscr{F}_{r}(M)$ is said to be locally stable at a compact leaf $c$ of $w_{0}$ if there exist neighborhoods $\mathscr{N}\left(w_{0}\right)$ of $w_{0}$ in $\mathscr{F}_{r}{ }^{1}(M)$ and $U(c)$ of $c$ in $M$ such that for each $w$ in $\mathscr{N}\left(w_{0}\right)$ there is a compact leaf $d=d(w)$ of $w$ in $U(c)$ and a homeomorphism $h: V(d) \rightarrow V(c)$ between neighborhoods of $d$ and $c$ such that $h(d)=c$ and such that $h$ maps the configuration of $w / V(d)$ onto the configuration of $w_{0} / V(c)$.

6.4. Proposition. Let $c$ be a hyperbolic compact leaf of $w$ in $\mathscr{F}_{r}(M)$. Then $w$ is locally stable at $c$.

For the proof of this proposition we shall need the following definition and remark. They will be used again later. 
6.5. Definition. (a) Let $w \in \mathscr{F}_{r}(M)$ and $U \subseteq M$ be an open set contained in $M-\operatorname{Sing}(w)$. A pair $\left(X_{1}, X_{2}\right)$ of $C^{r}$-vector fields is said to be fitted to $w$ in $U$ if $X_{i}(p)$ is tangent to $f_{i}(w)$ at $p$ for all $p \in U$ and $i=1,2$. Such a pair will be denoted by $\left(X_{1}(w), X_{2}(w)\right)$.

(b) Let $\left(X_{1}, X_{2}\right)$ be a pair of transversal $C^{r}$-vector fields defined in an open set $U$ of $M$. Consider the 1-forms defined by $\Theta_{i}(p)\left(X_{j}(p)\right)=1-\delta_{i j}$ for all $p \in U$ and $i, j=1,2$. Then the positive quadratic differential form $w=\Theta_{1} \cdot \Theta_{2}$ is said to be associated to $\left(X_{1}, X_{2}\right)$ in $U$ and denoted by $w\left(X_{1}, X_{2}\right)$.

6.6. REMARK. Let $w$ be in $\mathscr{F}_{r}(M)$, let $\gamma$ be a leaf of $f_{1}(w)$, and $\gamma_{0}$ be a compact arc of $\gamma$. Then:

(a) There exist an open neighborhood $U$ of $\gamma_{0}$ in $M$ and a pair $\left(X_{1}, X_{2}\right)$ of $C^{r}$-vector fields fitted to $w$ in $U$.

(b) There exists a $C^{r}$-function $\lambda: U \rightarrow \mathbf{R}-\{0\}$ such that $w / U=\lambda w\left(X_{1}, X_{2}\right)$ (Lemma 4.2).

(c) Given an open subset $V$ with $\bar{V} \subset U$ and a pair of transversal $C^{r}$-vector fields $\left(Y_{1}, Y_{2}\right)$ defined in $U$ such that $Y_{i}=X_{i}$ outside $V$, the quadratic differential form $\tilde{w}$ defined by $\tilde{w}=\lambda w\left(Y_{1}, Y_{2}\right)$ in $U$ and $\tilde{w}=w$ outside $U$ is $C^{r}$ and positive. That is, $\tilde{w} \in \mathscr{F}_{r}(M)$.

6.7. PROOF OF PROPOSITION 6.4. Take a smooth riemannian metric on $M$. Let $c$ be a hyperbolic compact leaf of a $w$ in $\mathscr{F}_{r}(M)$, say $c$ in $f_{1}(w)$. Let $U$ be an open neighborhood of $c$ in $M$ and $\mathscr{V}$ be an open neighborhood of $w$ in $\mathscr{F}_{r}{ }^{1}(M)$ such that $U \cap \operatorname{Sing}(\tilde{w})=\varnothing$ and the foliations $f_{1}(\tilde{w})$ and $f_{2}(\tilde{w})$ can be oriented in $U$ for all forms $\tilde{w} \in \mathscr{V}$. We orient $f_{1}(\tilde{w})$ and $f_{2}(\tilde{w})$ in $U$ in such a way that the functions $\tilde{w} \in \mathscr{V} \rightarrow X_{i}(\tilde{w})(p), i=1,2$, are continuous for all $p \in U$, where $\left(X_{1}(\tilde{w}), X_{2}(\tilde{w})\right)$ is the corresponding pair of unit $C^{r}$-vector fields fitted to $\tilde{w}$ in $U$. Since the function $\tilde{w} \in \mathscr{V} \rightarrow\left(X_{1}(\tilde{w}), X_{2}(\tilde{w})\right)$ is continuous and $c$ is a hyperbolic periodic orbit of $X_{1}(w)$, it follows from vector field theory that $w$ is locally stable at $c[\mathbf{7}$, p. 98].

6.8. THEOREM. The set $S_{r}(M)$ defined in $\S 3$ is open in $\mathscr{F}_{r}^{1}(M)$ and every $w \in S_{r}(M)$ is structurally stable.

PROOF. As we said, the openness of $S_{r}(M)$ in $\mathscr{F}_{r}^{1}(M)$ follows from the local stability of hyperbolic singular points and the continuity, on compact parts, under small $C^{1}$-perturbations of singular separatrices together with the local stability of hyperbolic compact leaves.

Also, the structural stability of $w \in S_{r}(M)$ follows from the canonical region method showed in [12, pp. 210-212].

7. Density of $S_{r}(M)$ in $G_{r}(M)$. In this section we will prove that $S_{r}(M)$ is dense in $G_{r}(M)$ with the $C^{s}$-topology for any $s \leq r$. For this consider the following open subsets of $\mathscr{F}_{r}^{1}(M): \mathscr{H}_{1}$ is the subset of $w$ in $G_{r}(M)$ whose singular points are all hyperbolics; $\mathscr{H}_{2}$ is the subset of those $w$ in $\mathscr{H}_{1}$ with associated foliations without nontrivial recurrent leaves; and $\mathscr{H}_{3}$ is the subset of those $w$ in $\mathscr{H}_{2}$ without double separatrices. We will prove that $\mathscr{H}_{1}$ is dense in $G_{r}(M)$, that $\mathscr{H}_{2}$ is dense in $\mathscr{H}_{1}$, that $\mathscr{H}_{3}$ is dense in $\mathscr{H}_{2}$, and that $S_{r}(M)$ is dense in $\mathscr{H}_{3}$.

7.1. PROPOSItION. The set $\mathscr{H}_{1}$ is dense in $G_{r}(M)$ with the $C^{s}$-topology for any $s \leq r$. 
PrOOF. Let $w$ be in $G_{r}(M), s \leq r$ and let $p$ be a singular point of $w$. Consider a local chart $(x, y):(U, p) \rightarrow\left(\mathbf{R}^{2}, 0\right)$ such that $\operatorname{Sing}(w) \cap U=\{p\}$ and $(x, y)^{*}(w)=$ $\left(y+M_{1}(x, y)\right) d y^{2}+\left(b_{1} x+b_{2} y+M_{2}(x, y)\right) d x d y+\left(-y+M_{3}(x, y)\right) d x^{2}$, with $b_{1} \neq 0$ and $M_{i}(x, y)=O\left(x^{2}+y^{2}\right), i=1,2,3$. Let $V_{1}, V_{2}$ be neighborhoods of $(0,0)$ such that $\bar{V}_{2}$ is compact and $\bar{V}_{1} \subset V_{2} \subset \bar{V}_{2} \subseteq(x, y)(U)$. Let $\phi: \mathbf{R}^{2} \rightarrow[0,1]$ be a smooth function such that $\phi^{-1}(1)=\bar{V}_{1}$ and $\phi^{-1}(0)=\mathbf{R}^{2}-V_{2}$. For $\delta \geq 0$ let $w_{\delta}$ be the positive $C^{r}$-quadratic differential form defined in $U$ by

$$
\begin{aligned}
w_{\delta}= & (x, y)_{*}\left(\left(y+M_{1}(x, y)\right) d y^{2}+(1+2 \delta \phi(x, y))\left(b_{1} x+b_{2} y+M_{2}(x, y)\right) d x d y\right. \\
& \left.+\left(-y+M_{3}(x, y)\right) d x^{2}\right) .
\end{aligned}
$$

Since the functions $g_{1}(\delta)=(1+2 \delta)^{2}\left(b_{2} / 2\right)^{2}+1-(1+2 \delta) b_{1}$ and $g_{2}(\delta)=1-(1+2 \delta) b_{1}$ are not vanishing for all small $\delta \neq 0$, there exists $\tilde{w}$ in $G_{r}(M)$ arbitrarily $C^{s}$-close to $w$ such that $\operatorname{Sing}(\tilde{w})=\operatorname{Sing}(w)$ and $p$ is a hyperbolic singular point of $\tilde{w}$.

Finally, since $w$ has only a finite number of singular points, the proposition is proved.

7.2. PROPOSITION. The set $\mathscr{H}_{2}$ of $w$ in $\mathscr{H}_{1}$ with associated foliations without nontrivial recurrent leaves is dense in $\mathscr{H}_{1}$ with the $C^{s}$-topology for any $s \leq r$.

PROOF. We will only prove that nontrivial recurrent leaves of $w$ in $\mathscr{H}_{1}$ can be eliminated in a way similar to the case of vector fields over orientable two-manifolds (see proof of Lemmas 2.4 and 2.5 of [7, pp. 144-150]).

Let $R$ be a small square contained in $M-\operatorname{Sing}(w)$ with horizontal sides $c a$ and $d b$ over $f_{1}(w)$ and vertical sides $c d$ and $a b$ over $f_{2}(w)$ as in Figure 4 and let $\left(X_{1}(w), X_{2}(w)\right)$ be the corresponding pair of $C^{r}$-unit vector fields fitted to $w$ in $R$.

Consider a point $p$ at the interior of $R$ and the leaves $\gamma_{1}$ of $f_{1}(w)$ and $\gamma_{2}$ of $f_{2}(w)$ passing through $p$. Suppose that there exist points $p_{1}, p_{2}$ in $\gamma_{1} \cap \gamma_{2} \cap \operatorname{int}(R)$ such that the oriented segments $p p_{1}$ (respectively $p p_{2}$ ) along $\gamma_{1}$ (resp. $\gamma_{2}$ ) have first intersection with boundary of $R$ at a point of $[a, b]$ (resp. $[c, d]$ ) and the last intersection with boundary of $R$ at a point of $[c, d]$ (resp. $[a, b]$ ) (see Figure 4). Observe that this situation must happen if we have a nontrivial recurrent leaf.

Let $X_{1}^{u}(w)=X_{1}(w)+\delta u Y$ be a family of $C^{r}$-vector fields where $\delta>0,0 \leq u \leq 1$, and $Y$ is a field that is transversal to $X_{1}(w)$ on the interior of $R$, points upwards, and vanishes outside $R$. If $\delta$ is small enough, $X_{1}^{u}(w)$ is near to $X_{1}(w)$ and is transversal

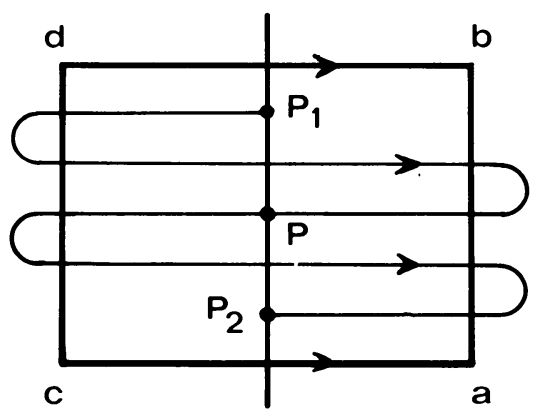

FIGURE 4 
to $X_{2}(w)$ for all $0 \leq u \leq 1$ and we can consider the family $\{w(u)\} \subseteq \mathscr{F}_{r}(M)$ defined by

$$
w(u)= \begin{cases}\lambda w\left(X_{1}^{u}(w), X_{2}(w)\right) & \text { on } R, \\ w & \text { on } M-R,\end{cases}
$$

where $\lambda: R \rightarrow \mathbf{R}-\{0\}$ is the $C^{r}$-function such that $w / R=\lambda w\left(X_{1}(w), X_{2}(w)\right)$.

Since the manifold is orientable, as in the case of vector fields, the functions $u \rightarrow p_{1}(u), u \rightarrow p_{2}(u)$ are monotone, one increasing and other decreasing on $\gamma_{2}$.

Therefore, our proposition can be proved in a way similar to the case of vector fields over orientable two-manifolds.

7.3. PROPOSITION. The set $\mathscr{H}_{3}$ of $w$ in $\mathscr{H}_{2}$ without double separatrices is dense in $\mathscr{H}_{2}$ with the $C^{s}$-topology for any $s \leq r$.

PROOF. Observe that any $w$ in $\mathscr{H}_{2}(M)$ has a finite number of singular points and therefore a finite number of double separatrices. This double separatrices can be broken by using Remark 6.6 creating new compact leaves.

7.4. REMARK. To establish the density of $S_{r}(M)$ in $\mathscr{H}_{3}$ and therefore in $G_{r}(M)$, it is sufficient to approximate any $w$ in $\mathscr{H}_{3}$ by $\tilde{w}$ in $\mathscr{H}_{3}$ with only a finite number of compact leaves, all of them hyperbolics. The proof is and adaptation of that in $[\mathbf{1}$, p. 114-122] for a similar result for line fields.

7.5. Definition. Let $w \in \mathscr{F}_{r}(M)$ and $i \in\{1,2\}$.

(a) A $C^{r}$-submanifold of $M$ contained in $M-\operatorname{Sing}(w)$ is said to be a "quasicylindrical region" of $f_{i}(w)$ if it is either a compact leaf of $f_{i}(w)$, a closed cylinder or a torus, and

(i) If it is a closed cylinder, its boundary consists of two compact leaves of $f_{i}(w)$.

(ii) If it is a torus, it contains a compact leaf of $f_{i}(w)$ not homotopic to a point. (In this case, the manifold is the torus.)

(b) If $\gamma$ is a compact leaf of $f_{i}(w)$ then $R_{i}^{*}(\gamma)$ will denote the set of all quasicylindrical regions of $f_{i}(w)$ that contain $\gamma$, and $R_{i}(\gamma)$ will denote the union of all elements of $R_{i}^{*}(\gamma)$.

(c) A quasi-cylindrical region $R$ is said to be a "maximal region" of $f_{i}(w)$ if there exists a compact leaf $\gamma$ of $f_{i}(w)$ such that $R_{i}(\gamma)=R$.

7.6. REMARK. If $w$ is in $\mathscr{H}_{3}$, then $f_{1}(w)$ and $f_{2}(w)$ have only a finite number of maximal regions, all of them compact.

7.7. Lemma. Let $w$ be in $\mathscr{H}_{3}$ and $\gamma$ be a compact leaf of $f_{1}(w)$. Then, given $s \leq r$, there exist $\tilde{w}$ in $\mathscr{H}_{3}$, arbitrarily $C^{s}$-close to $w$, and an open neighborhood $V$ of $\gamma$ such that $\tilde{w}=w$ outside $V$ and each compact leaf of $f_{1}(\tilde{w})$ contained in $V$ is hyperbolic.

PROOF. Follows from Remark 6.6 and the vector field theory [7, pp. 101-106].

7.8. Definition. Let $w \in \mathscr{F}_{r}(M)$ and $i \in\{1,2\}$. A quasi-cylindrical region $A$ of $f_{i}(w)$ is said to be a "Reeb component" of $f_{i}(w)$ if:

(a) $A$ is a closed cylinder.

(b) There are no compact leaves of $f_{i}(w)$ contained in the interior of $A$.

(c) There is no transversal section for $f_{i}(w)$ in $A$ joining the two circles of the boundary of $A$. 
For instance (see Figure 5):

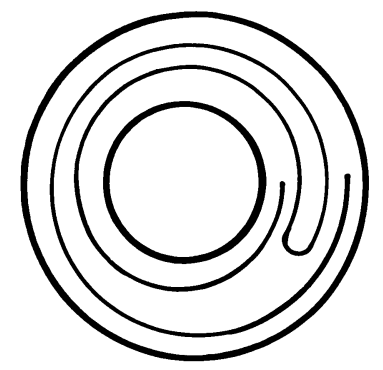

FIGURE 5

7.9. LeMMA. Let $w \in \mathscr{H}_{3}$ and $R$ be a quasi-cylindrical region of $f_{1}(w)$. Then $R$ contains a finite number of Reeb components of $f_{1}(w)$.

PROOF. Evident because $R$ is compact and at every regular point of $w$ there exists a tubular flow for $f_{1}(w)$; i.e., for each regular point $p$ there exists a small square $F$, with $p$ in $\operatorname{int}(F)$ and horizontal sides over $f_{1}(w)$ and such that each leaf of $f_{1}(w) / F$ meets both vertical sides at exactly one point.

7.10 LEMMA. Let $w$ be in $\mathscr{H}_{3}$ and $R$ be a maximal region of $f_{1}(w)$. Given $s \leq r$ and an open neighborhood $U$ of $R$ with $\bar{U} \subseteq M-\operatorname{Sing}(w)$ and no compact leaf of $f_{1}(w)$ meeting $\bar{U}-R$, there exists $\tilde{w}$ in $\mathscr{\mathscr { H }}_{3}$, arbitrarily $C^{s}$-close to $w$ with $\tilde{w}=w$ outside $U$ and such that every compact leaf of $f_{1}(\tilde{w})$ in $U$ is hyperbolic.

PROOF. Let $s \leq r$ and $U$ be an open neighborhood of $R$ as above. If $R$ is a compact leaf, it is enough to apply Lemma 7.7. If $R$ is a closed cylinder, let $A_{1}, \ldots, A_{n}$ be the Reeb components of $f_{1}(w)$ contained in $R$. So $R-\operatorname{int}\left(\bigcup_{i=1}^{n} A_{i}\right)=$ $\bigcup_{j=1}^{k} R_{j}$ with each $R_{j}$ a quasi-cylindrical region for $f_{1}(w)$. If some $R_{j}$ is a compact leaf, we apply Lemma 7.7. If $R_{j}$ is a closed cylinder it is possible to find a transversal for $f_{1}(w)$ and therefore it is possible to orient $f_{1}(w)$ over $R_{j}$. Since $f_{1}(w)$ and $f_{2}(w)$ are transversal we can also orient $f_{2}(w)$ over $R_{j}$. Now, we extend these orientations from a small neighborhood $V_{j}$ of $R_{j}$ and we consider the corresponding pair $\left(X_{1}(w), X_{2}(w)\right)$ of $C^{r}$-unit vector fields fitted to $w$ in $V_{j}$. So, the proof of our lemma for $R_{j}$ follows from Remark 6.6 and the vector field theory. As the neighborhoods $V_{j}$ can be chosen disjoint and contained in $U$, the lemma is proved in this case.

Finally, if $R$ is a torus (hence $R=M=T^{2}$ ), let $c$ be a compact leaf of $f_{1}(w)$ which is not homotopic to a point. We can suppose, using Lemma 7.7 if necessary, that $c$ is hyperbolic. If $c$ is the only compact leaf of $f_{1}(w)$, the lemma is proved. If not, we consider the maximal quasi-cylindrical region that does not contain $c$. This is either a compact leaf of $f_{1}(w)$ or else a closed cylinder. Therefore, we return to the earlier cases and the lemma is proved.

7.11. Proposition. The set $S_{r}(M)$ (defined in $\S 3$ ) is open and dense in $\mathscr{H}_{3}$ and therefore in $G_{r}(M)$ with the $C^{s}$-topology for any $s \leq r$.

ProOF. Follows from Lemma 7.10 and Propositions 7.1, 7.2, and 7.3.

8. Density of $G_{\infty}(M)$ in $\mathscr{F}_{\infty}^{2}(M)$. The density of $G_{\infty}(M)$ in $\mathscr{F}_{\infty}^{2}(M)$ (Theorem $\mathrm{B}(\mathrm{iii})$ ) follows immediately from the three propositions that we state below. 
Given $w$ in $\mathscr{F}_{\infty}(M)$ define:

(i) $M_{k+1}(w)=\left\{p \in M \mid j_{k}\left(\pi_{2} w\right)(p)=0\right\}$, where $\pi_{2}$ is the projection $\pi_{2}(p, \alpha)=\alpha$ and $j_{k}\left(\pi_{2} w\right)(p)=0$ means that if in coordinates $(x, y):(U, p) \rightarrow\left(\mathbf{R}^{2}, 0\right),(x, y)^{*}(w)$ $=a(x, y) d y^{2}+b(x, y) d x d y+c(x, y) d x^{2}$, then $j_{k} a(0)=j_{k} b(0)=j_{k} c(0)=0$.

(ii) $R_{0}(w)=\{p \in \operatorname{Sing}(w) \mid p$ is simple $\}$.

(iii) $R_{k}(w)=M_{k}(w)-M_{k+1}(w), k \geq 1$.

Given $s, k \in \mathbf{N}, k \leq s$, denote by $A(k, s)$ the following assertion: "For each $w$ in $\mathscr{F}_{\infty}(M)$ and each open subset $U \subseteq M$ such that $M_{k+1}(w) \subset U$, there exists $\tilde{w} \in$ $\mathscr{F}_{\infty}(M)$ arbitrarily $C^{s}$-close to $w$ such that $M_{k+1}(\tilde{w})=M_{k+1}(w)$ and $\operatorname{Sing}(\tilde{w}) \cap$ $(M-U) \subseteq R_{k-1}(\tilde{w}) "$.

8.1. Proposition. Let $s \in \mathbf{N}$. If the assertion $A(k, s)$ holds for all $k \leq s$, then the set $G_{\infty}(M)$ is dense in $\mathscr{F}_{\infty}^{s}(M)$.

8.2. Proposition. Let $w$ be in $\mathscr{F}_{\infty}(M)$ and $k$ in $\mathbf{N}$. Given $s \in \mathbf{N}, s \geq k$, and $U$ an open subset such that $M_{k+1}(w) \subset U$, there exists $\tilde{w}$ in $\mathscr{F}_{\infty}(M)$ arbitrarily $C^{s}$-close to $w$ such that $M_{k+1}(\tilde{w}) \subset U$ and $(M-\bar{U}) \cap R_{k}(\tilde{w})$ is a finite set.

8.3. PROPOSITION. Let $w$ be in $\mathscr{F}_{\infty}(M)$ and $p$ be an isolated point in $R_{2}(w)$ (respectively in $R_{1}(w)$ ). Then given $s \in \mathbf{N}, s \geq 2$ (resp. $s \geq 1$ ), there exist $\tilde{w}$ in $\mathscr{F}_{\infty}(M)$ arbitrarily $C^{s}$-close to $w$ and a neighborhood $V$ of $p$ such that $\tilde{w}=w$ outside $V$ and $V \cap \operatorname{Sing}(\tilde{w}) \subseteq R_{1}(\tilde{w})\left(\right.$ resp. $\left.V \cap \operatorname{Sing}(\tilde{w}) \subseteq R_{0}(\tilde{w})\right)$.

8.4. COROLLARY. The assertion $A(k, 2)$ holds for all $k \leq 2$.

8.5. REMARK. The problem of the $C^{s}$-density of $G_{\infty}(M)$ in $\mathscr{F}_{\infty}(M)$ for $s \geq 3$ is reduced to the problem of the generalization of Proposition 8.3 for any isolated point of $R_{k}(w)$, for all $k \leq s$.

In order to prove Proposition 8.1, we will use the following lemma whose proof can be found in $[\mathbf{1}$, p. 42$]$.

8.6. LEMMA. Let $d$ be a smooth riemannian metric on $M$. Then, given $s \in \mathbf{N}$, there exists a constant $A>1$ such that for every compact $K \subset M$, there exists $R>0$ such that for every $r, 0<r<R$, it is possible to find a smooth function $\phi_{r}: M \rightarrow[0,1]$ satisfying:

(i) $\phi_{r}^{-1}(1)$ is a neighborhood of $K$.

(ii) $\phi_{r}^{-1}(0)$ contains the set $\{p \in M \mid d(p, K) \geq r\}$.

(iii) $\forall j \in\{0,1, \ldots, s\},\left\|D^{j} \phi_{r}\right\| \leq A / r^{j}$.

PROOF OF PROPOSITION 8.1. Let $w$ be in $\mathscr{F}_{\infty}(M)$ and $\varepsilon>0$. Let $d$ be a smooth riemannian metric on $M$ and consider the constants $A>1$ and $R>0$ associated to $s$ and $M_{s+1}(w)$ in Lemma 8.6 above. For every point $p$ in $M_{s+1}(w)$, let $B_{p} \subset M$ be an open ball with center in $p$ and radius smaller than $R$ and such that $\|w / \bar{B}\|_{s}<\varepsilon / A 2^{s+3}$. Let $p_{1}, \ldots, p_{k}$ in $M_{s+1}(w)$ be such that $V=\bigcup_{i=1}^{k} B_{p_{i}} \supset$ $M_{s+1}(w)$ and $r$ be the distance between $M_{s+1}(w)$ and the boundary of $V$. Since $0<r<R$, there exists a smooth function $\phi: M \rightarrow[0,1]$ such that $\phi^{-1}(1)$ is a neighborhood of $M_{s+1}(w), \phi^{-1}(0)$ contains the set $\left\{p \in M \mid d\left(p, M_{s+1}(w)\right) \geq r\right\}$ and $\left\|D^{j} \phi\right\| \leq A / r^{j}$ for $j=0,1, \ldots, s$. Therefore $\phi^{-1}(1) \subset V$ and $M-V \subset \phi^{-1}(0)$. Let $U$ be an open subset of $M$ such that $M_{s+1}(w) \subset U \subset \phi^{-1}(1)$.

1. Since $A(k, s)$ holds for all $k \leq s$, there exists $w_{1}$ in $\mathscr{F}_{\infty}(M)$ arbitrarily $C^{s}$-close to $w$ such that $M_{s+1}\left(w_{1}\right)=M_{s+1}(w)$ and $\operatorname{Sing}\left(w_{1}\right) \cap(M-U) \subseteq R_{s-1}\left(w_{1}\right)$. Therefore $M_{s}\left(w_{1}\right) \subset U$ and there exists $w_{2}$ in $\mathscr{F}_{\infty}(M)$ arbitrarily $C^{s}$-close to $w_{1}$ such 
that $M_{s}\left(w_{2}\right)=M_{s}\left(w_{1}\right)$ and $\operatorname{Sing}\left(w_{2}\right) \cap(M-U) \subseteq R_{s-2}\left(w_{2}\right)$. Then, repeating this process $s$-times more we find a $w_{s}$ in $\mathscr{F}_{\infty}(M)$ such that $M_{s+1}\left(w_{s}\right)=M_{s+1}(w) \subset U$, $\operatorname{Sing}\left(w_{s}\right) \cap(M-U)=\left\{q_{1}, \ldots, q_{n}\right\} \subset R_{0}\left(w_{s}\right)$ and $\left\|w_{s}-w\right\|_{s}<\varepsilon / A 2^{s+3}$. Since $\left\|w_{s} / \bar{V}\right\|_{s}<\varepsilon / A 2^{s+2}$ we also have $\left\|\phi w_{s}\right\|_{s}<\varepsilon / 4$ (Mean Value Theorem).

2. Observe that any $\tilde{w}$ in $\mathscr{F}_{\infty}(M)$ with $\|\tilde{w}\|_{s}$ small enough and $\operatorname{Sing}(\tilde{w}) \cap \bar{U} \subseteq$ $R_{0}(\tilde{w})$ does not solve our problem because the $C^{\infty}$-quadratic differential form $\tilde{w}+$ $(1-\phi) w_{s}$ is not necessarily positive.

3. To find an appropriate $\tilde{w}$ we extend in a suitable way the foliations $f_{1}\left(w_{s}\right) / M-$ $U$ and $f_{2}\left(w_{s}\right) / M-U$ to each connected component of $U$ and then we use our Realization Theorem (Theorem A). For this note that we can suppose that $U$ has only a finite number of connected components with boundary a finite union of closed regular smooth curves that are disjoint from the set $\operatorname{Sing}\left(w_{s}\right)$. Also we can suppose that the foliations $f_{1}\left(w_{s}\right)$ and $f_{2}\left(w_{s}\right)$ have only a finite number of tangencies, all of parabolic type, with these boundaries.

4. For each connected component $U_{j}$ of $U$, let $a_{1}(i), \ldots, a_{l_{i}}(i)$ (resp. $b_{1}(i), \ldots$, $\left.b_{m_{i}}(i)\right)$ be the points of external (resp. internal) parabolic tangency of the foliation $f_{i}\left(w_{s}\right)$ with the boundary $\mathscr{T}_{j}$ of $U_{j}$. Then, we have $l_{1}-m_{1}=l_{2}-m_{2}$ and the number $c_{j}=\frac{1}{2}\left(2-l_{1}+m_{1}\right)$ is the Poincaré index of $\mathscr{T}_{j}$ for $f_{1}\left(w_{s}\right)$ and $f_{2}\left(w_{s}\right)$.

5. Now, we choose some positive $C^{\infty}$-quadratic differential forms $\Theta_{1}, \ldots, \Theta_{m}$ defined respectively on small open and disjoint balls $B_{1}, \ldots, B_{m}$ contained in $U$ such that:

(a) Each $\Theta_{k}$ has a unique singular point $p_{k}$ which is simple.

(b) At each connected component $U_{j}$ of $U$ the sum of the Poincaré index of these singular points is $c_{j}$.

(c) There exist transversal smooth foliations $\tilde{f}_{1}, \tilde{f}_{2}$ defined in $M-\left\{q_{1}, \ldots, q_{n}\right.$, $\left.p_{1}, \ldots, p_{m}\right\}$ such that $\tilde{f}_{i} / M-U=f_{i}\left(w_{s}\right)$ and $\tilde{f}_{i} / B_{k}=f_{i}\left(\Theta_{k}\right)$ for all $k=1, \ldots, m$ and $i=1,2$.

6. Let $\tilde{w}$ in $\mathscr{F}_{\infty}(M)$ be such that $C(\tilde{w})=\left\{\tilde{f}_{1}, \tilde{f}_{2},\left\{q_{1}, \ldots, q_{n}, p_{1}, \ldots, p_{m}\right\}\right\}$ (Theorem A).

7. Let $\psi: M \rightarrow[0,1]$ be a smooth function such that $\psi^{-1}(1)$ is a neighborhood of $\left\{p_{1}, \ldots, p_{m}\right\}$ and $\psi^{-1}(0)=M-\bigcup_{k=1}^{m} V_{k}$ with $V_{k}$ an open ball centered at $p_{k}$ and $\bar{V}_{k} \subset B_{k}$ for all $k=1, \ldots, m$. (Therefore $\psi^{-1}(1) \subset \bigcup_{k=1}^{m} V_{k}$.)

8. Let $\tilde{w}_{\lambda}$ be the smooth quadratic differential form defined by $\check{w}_{\lambda}=(1-\phi) w_{s}+$ $\lambda \phi \tilde{w}$ outside of $\bigcup_{k=1}^{m} B_{k}$ and by $\check{w}_{\lambda}=\lambda\left[(1-\psi) \tilde{w}+\psi \tilde{\Theta}_{k}\right]$ on each $B_{k}$ with $\lambda>0$. Then $\tilde{w}_{\lambda}$ is positive because the corresponding forms have the same configuration where $\phi$ or $\psi$ are not vanishing.

Then $\tilde{w}_{\lambda} \in \mathscr{F}_{\infty}(M)$ and $\operatorname{Sing}\left(\tilde{w}_{\lambda}\right)=\left\{q_{1}, \ldots, q_{n}, p_{1}, \ldots, p_{m}\right\}$. Clearly $p_{1}, \ldots, p_{m}$ and the $q_{i}$ 's that are in $\phi^{-1}(0)$ are simple. The other $q_{i}$ 's are also simple because $\tilde{w}$ is flat at each singular point (see proof of Theorem A).

Therefore $\tilde{w}_{\lambda} \in G_{\infty}(M)$ and if $\lambda>0$ is small enough we have $\left\|\tilde{w}_{\lambda}-w_{s}\right\|_{s}<\varepsilon / 2$ and then $\left\|\tilde{w}_{\lambda}-w\right\|_{s}<\varepsilon$. Thus the proof of Proposition 8.1 is complete.

The proofs of Propositions 8.2 and 8.3 will be given in $\S \S 10$ and 11 respectively, using some preliminary lemmas that we introduce in the next section.

9. Technical lemmas. In this section we state some technical lemmas that are used in the proofs of Propositions 8.2 and 8.3 ( $\$ \S 10$ and 11).

In order to simplify we introduce the following sets. 
If $U$ is an open subset of $\mathbf{R}^{2}$, let $A_{r}(U)$ be the set of triples $(a, b, c)$ of realvalued functions defined in $U$ such that the quadratic differential form $a(x, y) d y^{2}+$ $b(x, y) d x y+c(x, y) d x^{2}$ is in $\mathscr{F}_{r}(U)$. That is, the functions $a, b, c$ are of class $C^{r}$, $\left(b^{2}-4 a c\right) \geq 0$ in $U$, and $\left(b^{2}-4 a c\right)^{-1}(0)=a^{-1}(0) \cap b^{-1}(0) \cap c^{-1}(0)$.

If $U_{1}, U_{2}$ are open subsets of $\mathbf{R}^{2}$ with $\bar{U}_{1} \subset U_{2}$ and $\bar{U}_{2}$ a compact set, let $b\left(U_{1}, U_{2}\right)$ be the set of smooth functions $\phi: \mathbf{R}^{2} \rightarrow[0,1]$ such that $\phi^{-1}(1)=\bar{U}_{1}$ and $\phi^{-1}(0)=\mathbf{R}^{2}-U_{2}$.

9.1. LEMMA. Let $U$ be an open neighborhood of $(0,0)$ in $\mathbf{R}^{2}$ and $(a, b, c)$ be a triple in $A_{r}(U)$. Suppose that $\left(\partial^{k} / \partial x^{i} \partial y^{k-i}\right)(a, b, c)(0,0) \neq(0,0,0)$ for some $0 \leq k \leq r$ and $0 \leq i \leq k$. Then given $s \in \mathbf{N}, s \leq r, \varepsilon>0$, and an open neighborhood $V$ of $(0,0)$ contained in $U$, there exist $(\tilde{a}, \tilde{b}, \tilde{c}) \in A_{r}(U)$ such that:

(a) $\left(\tilde{b}^{2}-4 \tilde{a} \tilde{c}\right)^{-1}(0)=\left(b^{2}-4 a c\right)^{-1}(0)$.

(b) $(\tilde{a}, \tilde{b}, \tilde{c})=(a, b, c)$ outside $V$.

(c)

$$
\frac{\partial^{k} \tilde{a}}{\partial x^{i} \partial y^{k-i}}(0,0) \cdot \frac{\partial^{k} \tilde{b}}{\partial x^{i} \partial y^{k-i}}(0,0) \cdot \frac{\partial^{k} \tilde{c}}{\partial x^{i} \partial y^{k-i}}(0,0) \neq 0 .
$$

(d) $\max \left\{\|\tilde{a}-a\|_{s},\|\tilde{b}-b\|_{s},\|\tilde{c}-d\|_{s}\right\}<\varepsilon$.

Proof. Let $s \in \mathbf{N}, s \leq r, \varepsilon>0$ and $V$ be an open neighborhood of $(0,0)$ contained in $U$. Consider $\Theta \in b\left(U_{1}, U_{2}\right)$ with $(0,0) \in U_{1}$ and $\bar{U}_{2} \subset V$. Then if $\left(\partial^{k} / \partial x^{i} \partial y^{k-i}\right)(a, b, c)(0,0)=\left(a_{0}, b_{0}, c_{0}\right)$, for $\delta>0$ we define

$$
(\tilde{a}, \tilde{b}, \tilde{c})=\left\{\begin{array}{c}
(a, b, c)+\delta \Theta(-b, 2(a-c), b) \quad \text { if }\left[b_{0} \neq 0 \text { or } b_{0}=0\right. \text { and } \\
\left.a_{0} \cdot c_{0} \neq 0 \text { and } a_{0} \neq c_{0}\right] \\
(a, b, c)+\delta \Theta(0,2 a, b) \quad \text { if } b_{0}=0 \text { and } a_{0}=c_{0}, \\
(a, b, c)+\delta \Theta(0,2 a, b+\delta \Theta a) \quad \text { if } b_{0}=c_{0}=0 \\
(a, b, c)+\delta \Theta(b+\delta \Theta c, 2 c, 0) \quad \text { if } b_{0}=a_{0}=0 .
\end{array}\right.
$$

Therefore $(\tilde{a}, \tilde{b}, \tilde{c}) \in A_{r}(U)$ and verifies conditions (a) and (b) and if $\delta>0$ is small enough also verifies conditions (c) and (d).

9.2. Lemma. Let $(a, b, c)$ be a triple in $A_{r}(U)$. Then given $s \in \mathbf{N}, s \leq r$, $\varepsilon>0$, and any open set $V$ of $\mathbf{R}^{2}$ with $\bar{V}$ a compact set contained in $U$, there exists a triple $(\tilde{a}, \tilde{b}, \tilde{c})$ in $A_{r}(U)$ such that:

(a) $(\tilde{a}, \tilde{b}, \tilde{c})=(a, b, c)$ outside $V$.

(b) $\left(\tilde{b}^{2}-4 \tilde{a} \tilde{c}\right)^{-1}(0)=\left(b^{2}-4 a c\right)^{-1}(0)$.

(c) If $(x, y)$ is in $V,\left(\partial^{k} / \partial y^{k}\right)(a, b, c)(x, y) \neq(0,0,0)$ and

$$
\left(\left(\frac{\partial^{k} b}{\partial y^{k}}\right)^{2}-4 \frac{\partial^{k} a}{\partial y^{k}} \cdot \frac{\partial^{k} c}{\partial y^{k}}\right)(x, y) \geq 0
$$

for some $k \leq r$ we have

$$
\left(\left(\frac{\partial^{k} \tilde{b}}{\partial y^{k}}\right)^{2}-4 \frac{\partial^{k} \tilde{a}}{\partial y^{k}} \cdot \frac{\partial^{k} \tilde{c}}{\partial y^{k}}\right)(x, y)>0 .
$$

(d) $\max \left\{\|\tilde{a}-a\|_{s},\|\tilde{b}-b\|_{s},\|\tilde{c}-c\|_{s}\right\}<\varepsilon$. 
PROOF. Let $s \in \mathbf{N}, s \leq r, \varepsilon>0$ and $V$ be an open set such that $\bar{V}$ is a compact set contained in $U$. Let $\phi \in b\left(V_{1}, V\right)$ and $\delta>0$ such that $\delta \cdot \max \left\{\|\phi \cdot(a-c)\|_{s}\right.$, $\left.\|2 \phi \cdot b\|_{s}\right\}<\varepsilon$. Then the triple $(\tilde{a}, \tilde{b}, \tilde{c})=(a, b, c)+\delta \phi(a-c, 2 b, c-a)$ belongs to $A_{r}(U)$ and satisfies (a), (b), (c), and (d).

9.3. LEMMA. Given $r>0$, for all $k \in \mathbf{N}$ there exists a smooth function $\psi_{k}: \mathbf{R} \rightarrow[0,1]$ such that:

$$
\begin{aligned}
& \psi_{k}^{-1}(1)=[-r, r],\left.\psi_{k}^{-1}(0)=\mathbf{R}-\right]-2 r, 2 r[ \\
&\left|\psi_{k}^{(i)}(x)\right| \leq 2^{i(i+1) / 2} \cdot r^{-i} \quad \text { for all } x \in \mathbf{R} \text { and } i=1, \ldots, k .
\end{aligned}
$$

PROOF. Let $r>0$. It is sufficient to prove that for all $k \in \mathbf{N}$, there exists a smooth function $\Theta_{k}: \mathbf{R} \rightarrow[0,1]$ such that: $\left.\left.\Theta_{k}^{-1}(0)=\right]-\infty, 0\right], \Theta_{k}^{-1}(1)=[r,+\infty[$, and $\left|\Theta_{k}^{(i)}(x)\right| \leq 2^{i(i+1) / 2} \cdot r^{-i}$ for all $x \in \mathbf{R}$ and $i=1, \ldots, k$.

To obtain $\Theta_{k}$ we see by induction that for all $k \in \mathbf{N}$ there exists a smooth function $f_{k}: \mathbf{R} \rightarrow[0,1]$ such that: $\left.\left.f_{k}^{-1}(0)=\right]-\infty, 0\right] ; f_{k}^{-1}(1)=\left[2^{k},+\infty\right] ;\left|f_{k}^{(i)}(x)\right| \leq$ $2^{i(i+1-2 k) / 2}$ for all $i=1, \ldots, k ; f_{k}(x)=1-f_{k}\left(2^{k}-x\right)$ and therefore $\int_{0}^{2^{k}} f_{k}(d) d x=$ $2^{k-1}$.

If we define $\Theta_{k}(x)=f_{k}\left(2^{k} \cdot r^{-1} x\right)$ the lemma is proved.

9.4. COROLlaRY. For $r>0$ let us consider the rectangles $R_{1}=\{(x, y)|| x \mid \leq$ $r,|y| \leq r\}$ and $R_{2}=\{(x, y)|| x|\leq 2 r| y \mid, \leq 2 r\}$. Then given $s \in \mathbf{N}$ there exists $\phi_{s} \in b\left(\operatorname{int} R_{1}\right.$, int $\left.R_{2}\right)$ such that $\left|\left(\partial^{i} \phi_{s} / \partial x^{j} \partial y^{i-j}\right)(x, y)\right| \leq 2^{i(i+1) / 2} \cdot r^{-i}$ for all $(x, y) \in \mathbf{R}^{2}, i=1, \ldots, s, j=0,1, \ldots, i$.

PROOF. For $r>0$ and $s \in \mathbf{N}$, it is enough to define $\phi_{s}(x, y)=\psi_{s}(x) \cdot \psi_{s}(y)$ for all $(x, y) \in \mathbf{R}^{2}$ with $\psi_{s}$ from the previous lemma.

9.5. REMARK. If we have $r>0, s \in \mathbf{N}$, and a $C^{s}$-function $f: \mathbf{R} \rightarrow \mathbf{R}$ (resp. $f: \mathbf{R}^{2} \rightarrow \mathbf{R}$ ) such that $f^{(i)}(p)=0$ (resp. $\left(\partial^{i} f / \partial x^{j} \partial y^{i-j}\right)(0,0)$ for all $j=$ $0,1, \ldots, i)$ for $i=0,1, \ldots, s$ and $\left\|f /{ }_{[-2 r, 2 r]}\right\|_{s}<\varepsilon / \lambda_{s}$ (resp. $\left.\left\|f_{R_{2}}\right\|_{s}<\varepsilon / \lambda_{s}\right)$ where $\varepsilon>0$ and $\lambda_{s}=2^{s} \cdot \sum_{i=1}^{s}\left(\begin{array}{l}s \\ i\end{array}\right) 2^{(s-i) \cdot(s-i+1) / 2}$, then it follows from the Mean Value Theorem that $\left\|\psi_{s} \cdot f\right\|_{s}<\varepsilon$ (resp. $\left\|\phi_{s} \cdot f\right\|_{s}<\varepsilon$ ) where $\psi_{s}$ (resp. $\phi_{s}$ ) is the function of Lemma 9.3 (resp. Corollary 9.4).

10. Proof of Proposition 8.2. The proof of Proposition 8.2. will be a consequence of some lemmas.

10.1. LEMMA. Let $w \in \mathscr{F}_{\infty}(M)$ and $I$ be a compact segment of a smooth curve in $M$. Suppose there exists an open set $V$ containing $I$ such that $V \cap R_{k}(w)=I$. Then, given $s \in \mathbf{N}$ there exists $\tilde{w} \in \mathscr{F}_{\infty}(M)$ arbitrarily $C^{s}$-close to $w$ such that $\tilde{w}=w$ outside $V$ and $V \cap R_{k}(w)$ is a finite set.

PRoOF. Let $s \in \mathbf{N}$ and $(x, y): U \subseteq M \rightarrow \mathbf{R}^{2}$ be a chart such that $I \subset U \subset$ $\bar{U} \subset V$ with $\bar{U}$ compact and $(x, y)(I)=J \times\{0\}$ and we suppose $J$ not a singleton.

If $(x, y)^{*}(w)=a(x, y) d y^{2}+b(x, y) d x d y+c(x, y) d x^{2}$, it follows from the Mather Division Theorem [6, p. 104] that there exist smooth functions $M_{1}, M_{2}, M_{3}, h_{i}, k_{i}, g_{i}$, $i=1, \ldots, k$, defined in $(x, y)(U)$ such that

$$
(a, b, c)(x, y)=y^{k}\left(M_{1}, M_{2}, M_{3}\right)+\sum_{i=1}^{k} y^{k-i}\left(h_{i}(x), k_{i}(x), g_{i}(x)\right)
$$

in $(x, y)(U)$. 
Then for all $x \in J,\left(h_{i}, k_{i}, g_{i}\right)(x)=(0,0,0)$ and $\left(M_{1}, M_{2}, M_{3}\right)(x, 0) \neq(0,0,0)$ and we can suppose that $M_{1}(x, 0) \cdot M_{2}(x, 0) \cdot M_{3}(x, 0) \neq 0$ for all $x \in J$ (Lemma 9.1).

If $J=\left[x_{0}, x_{1}\right]$ consider the following rectangles: $R_{1}=\left[x_{0}+r, x_{1}-r\right] \times[-r, r]$ and $R_{2}=\left[x_{0}, x_{1}\right] \times[-2 r, 2 r]$ with $r>0$ small enough to have $x_{0}+r<x_{1}-r$ and $M_{1}(x, y) \cdot M_{2}(x, y) \cdot M_{3}(x, y) \neq 0$ for all $(x, y) \in R_{2}$.

Let $\phi \in b\left(\right.$ int $R_{1}$, int $\left.R_{2}\right)$. Define

$$
(\tilde{a}, \tilde{b}, \tilde{c})= \begin{cases}(a, b, c)+\delta \phi\left(M_{1}, 0,-M_{3}\right) & \text { if } M_{1}\left(x_{0}, 0\right) M_{3}\left(x_{0}, 0\right)>0 \\ (a, b, c)+\delta \phi\left(0, M_{2}, 0\right) & \text { if } M_{1}\left(x_{0}, 0\right) M_{3}\left(x_{0}, 0\right)<0\end{cases}
$$

with $\delta \neq 0$.

Then in both cases $(\tilde{a}, \tilde{b}, \tilde{c}) \in A_{\infty}(U),(\tilde{a}, \tilde{b}, \tilde{c})=(a, b, c)$ outside $R_{2}$ and $R_{2} \cap$ $\left(\tilde{b}^{2}-4 \tilde{a c}\right)^{-1}(0)=\left\{\left(x_{0}, 0\right),\left(x_{1}, 0\right)\right\}$. Therefore if $\delta \neq 0$ is small enough, $w_{\delta}=$ $(x, y)_{*}\left(\tilde{a}(x, y) d y^{2}+\tilde{b}(x, y) d x d y+\tilde{c}(x, y)\right)$ verifies our lemma.

10.2. Lemma. Let $w \in \mathscr{F}_{\infty}(M)$ and $p \in R_{k}(w), k \geq 1$. Suppose that there exists a sequence $\left\{p_{n}\right\}_{n \in \mathbf{N}}$ contained in $R_{k}(w)-\{p\}$ converging to $p$. Then given $s \in \mathbf{N}$ there exists a neighborhood $V$ of $p$ and $\tilde{w} \in \mathscr{F}_{\infty}(M)$ arbitrarily $C^{s}$-close to $w$ such that $\tilde{w}=w$ outside $V$ and $R_{k}(\tilde{w}) \cap V$ is the union of a finite set and a compact segment of a smooth curve containing $p$.

Proof. Let $s \in \mathbf{N}$ and $(x, y):(U, p) \rightarrow\left(\mathbf{R}^{2},(0,0)\right)$ be a local chart with $y\left(p_{i}\right)=0$ for $i$ large enough. Let $(x, y)^{*}(w)=a(x, y) d y^{2}+b(x, y) d x d y+c(x, y) d x^{2}$.

Since $p \in R_{k}$ we have $\left(\partial^{k} / \partial y^{k}\right)(a, b, c)(0,0) \neq(0,0,0)$.

Therefore, we can suppose, using Lemma 9.2 if necessary, that

$$
\left(\left(\frac{\partial^{k} b}{\partial y^{k}}\right)^{2}-\frac{\partial^{k} a}{\partial y^{k}} \cdot \frac{\partial^{k} c}{\partial y^{k}}\right)(0,0)>0 \quad \text { and } \quad \frac{\partial^{k} a}{\partial y^{k}}(0,0) \cdot \frac{\partial^{k} b}{\partial y^{k}}(0,0) \cdot \frac{\partial^{k} c}{\partial y^{k}}(0,0) \neq 0 .
$$

Then, the $C^{\infty}$-Weierstrass Preparation Theorem [11, p. 152] implies that there exist an open neighborhood $V_{1} \subset(x, y)(U)$ of $(0,0)$ and smooth functions $M_{1}, M_{2}$, $M_{3}, h_{i}, k_{i}, g_{i}$, for $i=1, \ldots, k$, defined in $V_{1}$ such that

$$
\begin{aligned}
& a(x, y)=\left(y^{k}+\sum_{i=1}^{k} y^{k-i} h_{i}(x)\right) M_{1}(x, y), \\
& b(x, y)=\left(y^{k}+\sum_{i=1}^{k} y^{k-i} k_{i}(x)\right) M_{2}(x, y)
\end{aligned}
$$

and

$$
c(x, y)=\left(y^{k}+\sum_{i=1}^{k} y^{k-i} g_{i}(x)\right) M_{3}(x, y) \quad \text { for all }(x, y) \in V_{1} .
$$

So $\left(M_{2}^{2}-4 M_{1} M_{3}\right)(0,0)>0, M_{i}(0,0) \neq 0$ for $i=1,2,3$ and $h_{i}^{(n)}(0)=k_{i}^{(n)}(0)=$ $g_{i}^{(n)}(0)=0$ for all $n \in \mathbf{N}$ and $i=1, \ldots, k$.

Let $\varepsilon>0$. Let $V_{2}$ be an open neighborhood of $(0,0)$ such that $\bar{V}_{2}$ is a compact set contained in $V_{1} ; M_{i}(x, y) \neq 0$ for $i=1,2,3$ and $\left(M_{2}^{2}-4 M_{1} M_{3}\right)(x, y)>0$ for 
all $(x, y) \in V_{2}$, and

(*) $\quad \max \left\{\left\|(H+G-2 K) M_{1}\right\|_{s},\left\|(H-G) M_{1}\right\|_{s},\left\|(K-G) M_{1}\right\|_{s}\right.$,

$$
\left.\left\|(K-G) M_{3}\right\|_{s}\right\}<\varepsilon / 4 \lambda_{s} 3^{s}
$$

on $\bar{V}_{2}$ where $\lambda_{s}$ is as in Remark 9.5 and

$$
(H, K, G)(x, y)=\sum_{i=1}^{k} y^{k-i}\left(h_{i}(x), k_{i}(x), g_{i}(x)\right) .
$$

Consider the following rectangles: $R_{1}=\left\{(x, y)|| x\left|\leq \frac{1}{3} r,\right| y \mid \leq \frac{1}{3} r\right\}, R_{2}=$ $\left\{(x, y)|| x\left|\leq \frac{2}{3} r,\right| y \mid \leq \frac{2}{3} r\right\}$, and $R_{3}=\{(x, y)|| x|\leq r| y \mid, \leq r\}$ with $r>0$ small enough to have $R_{3} \subset V_{2}$.

Let $\phi_{1} \in b\left(\operatorname{int} R_{2}\right.$,int $\left.R_{3}\right)$ and $\phi_{2} \in b\left(\operatorname{int} R_{1}\right.$,int $\left.R_{2}\right)$ such that

$$
\left|\frac{\partial^{i} \phi_{k}}{\partial x^{j} \partial y^{i-j}}(x, y)\right| \leq 2^{i(i+1) / 2} \cdot(r / 3)^{-i} \quad \text { for all }(x, y) \in \mathbf{R}^{2} \text {, }
$$

$k=1,2, i=1,2, \ldots, s$, and $j=0,1, \ldots, i$ (Corollary 9.4).

It is sufficient to prove that there exists $(\tilde{a}, \tilde{b}, \tilde{c}) \in A_{\infty}\left(V_{2}\right)$ such that:

(1) $(\tilde{a}, \tilde{b}, \tilde{c})=(a, b, c)$ outside int $R_{3}$,

(2) $\max \left\{\|\tilde{a}-a\|_{s},\|\tilde{b}-b\|_{s},\|\tilde{c}-c\|_{s}\right\}<\varepsilon$,

(3) $(\tilde{a}, \tilde{b}, \tilde{c})(x, y)=\left(y^{k}-K(x, y)\right) \cdot\left(M_{1}, M_{2}, M_{3}\right)(x, y)$ for all $(x, y) \in R_{1}$,

(4) $\left(\tilde{b}^{2}-4 \tilde{a} \tilde{c}\right)^{-1}(0) \cap\left(V_{2}-R_{1}\right)=\left(b^{2}-4 a c\right)^{-1}(0) \cap\left(V_{2}-R_{1}\right)$.

Let us consider the cases $M_{1}(0,0) \cdot M_{3}(0,0)>0$ and $M_{1}(0,0) \cdot M_{3}(0,0)<0$ separately.

Case A: $M_{1}(0,0) \cdot M_{3}(0,0)>0$.

Let

$(\bar{a}, \bar{b}, \bar{c})(x, y)=(a, b, c)(x, y)+\phi_{1}(x, y)(H(x, y)+G(x, y)-2 K(x, y)) \cdot\left(M_{1}(x, y), 0,0\right)$.

Then

$$
\begin{aligned}
\left(\bar{b}^{2}-4 \bar{a} \bar{c}\right)(x, y)=\left(1-\phi_{1}(x, y)\right)\left(b^{2}-4 a c\right)(x, y)+\phi_{1}(x, y) & \\
\cdot\left[\left(y^{k}-K(x, y)\right)^{2}\right. & \left(M_{2}^{2}-4 M_{1} M_{3}\right)(x, y) \\
& \left.+4 M_{1}(x, y) M_{3}(x, y) \cdot(K(x, y)-G(x, y))^{2}\right] .
\end{aligned}
$$

Therefore, $(\bar{a}, \bar{b}, \bar{c}) \in A_{\infty}\left(V_{2}\right)$ and estimates $(*),(* *)$ and Remark 9.5 imply that $\|\bar{a}-a\|_{s}<\varepsilon / 2$.

Now let

$$
\left.(\tilde{a}, \tilde{b}, \tilde{c})(x, y)=(\bar{a}, \bar{b}, \bar{c})(x, y)+\phi_{2}(x, y)(K(x, y)-G(x, y)) \cdot\left(M_{1}, 0,-M_{3}\right)(x, y)\right) \text {. }
$$

Then for all $(x, y) \in R_{2}$ we have

$$
\begin{aligned}
\left(\tilde{b}^{2}-4 \tilde{a} \tilde{c}\right)(x, y)= & \left(y^{k}-K(x, y)\right)^{2}\left(M_{2}^{2}-4 M_{1} M_{3}\right)(x, y) \\
& \left.+4\left(1-\phi_{2}(x, y)\right)^{2}(K(x, y)-G(x, y))^{2} M_{1}(x, y) \cdot M_{3}(x, y)\right) .
\end{aligned}
$$

So $(\tilde{a}, \tilde{b}, \tilde{c}) \in A_{\infty}\left(V_{2}\right)$ and satisfies (1), (2), (3), and (4).

Case B. $M_{1}(0,0) M_{3}(0,0)<0$.

Let

$$
(\bar{a}, \bar{b}, \bar{c})(x, y)=(a, b, c)(x, y)+\phi_{1}(x, y)(H(x, y)-G(x, y))\left(M_{1}(x, y), 0,0\right) .
$$


Then

$$
\begin{aligned}
\left(\bar{b}^{2}-4 \bar{a} \bar{c}\right)(x, y)= & \left(1-\phi_{1}(x, y)\right)\left(b^{2}-4 a c\right)(x, y)+\phi_{1}(x, y) \\
& \cdot\left[\left(y^{k}-K(x, y)\right)^{2} M_{2}^{2}(x, y)-4\left(y^{k}-G(x, y)\right)^{2} M_{1}(x, y) M_{3}(x, y)\right]
\end{aligned}
$$

Therefore $(\bar{a}, \bar{b}, \bar{c})(x, y) \in A_{\infty}\left(V_{2}\right)$ and $\|\bar{a}-a\|_{s}<\varepsilon / 2$.

Now let

$$
(\tilde{a}, \tilde{b}, \tilde{c})(x, y)=(\bar{a}, \bar{b}, \bar{c})(x, y)+\phi_{2}(x, y)(G(x, y)-K(x, y)) \cdot\left(M_{1}, 0, M_{3}\right)(x, y) \text {. }
$$

Then for all $(x, y) \in R_{2}$ we have

$$
\begin{aligned}
\left(\tilde{b}^{2}-4 \tilde{a} \tilde{c}\right)(x, y)= & \left(y^{k}-K(x, y)\right)^{2} M_{2}^{2}(x, y) \\
& -4 \cdot\left[y^{k}-G(x, y)+\phi_{2}(x, y)(G(x, y)-K(x, y))\right]^{2} \\
& \cdot M_{1}(x, y) M_{3}(x, y) .
\end{aligned}
$$

So $(\tilde{a}, \tilde{b}, \tilde{c}) \in A_{\infty}\left(V_{2}\right)$ and satisfies (1), (2), (3), and (4).

10.3. LemMA (PROPOSITION 8.2). Let $k \in \mathbf{N}, w \in \mathscr{F}_{\infty}(M)$ and $V$ be an open subset of $M$ such that $\mathscr{M}_{k+1}(w) \subset M-\bar{V}$. Then, given $s \in \mathbf{N}, s>k$, there exists $\tilde{w} \in \mathscr{F}_{\infty}^{s}(M)$ arbitrarily close to $w$ such that $\mathscr{M}_{k+1}(\tilde{w}) \subset M-\bar{V}$ and $V \cap R_{k}(\tilde{w})$ is a finite set.

PROOF. Since $\mathscr{M}_{k+1}(w) \subset M-\bar{V}, V \cap R_{k}(w)$ is contained at the union of a finite number of disjoint smooth curves and we can suppose they are transversal to the boundary of $V$. If $I$ is one of these curves, consider a chart $(x, y): U \subseteq M \rightarrow \mathbf{R}^{2}$ such that $I \subset U, \mathscr{M}_{k+1}(w) \subset M-\bar{U}$ and $y(p)=0$ for all $p \in I$. Then if $(x, y)^{*}(w)=$ $a(x, y) d y^{2}+b(x, y) d x d y+c(x, y) d x^{2}$, it follows from the Mather Division Theorem that there exist smooth functions $M_{1}, M_{2}, M_{3}, h_{j}, k_{j}, g_{j}, j=1, \ldots, k$, defined in $(x, y)(U)$ such that

$$
a(x, y)=y^{k} M_{1}(x, y)+H(x, y), \quad b(x, y)=y^{k} M_{2}(x, y)+G(x, y)
$$

and

$$
c(x, y)=y^{k} M_{3}(x, y)+G(x, y)
$$

where

$$
(H, K, G)(x, y)=\sum_{j=1}^{k} y^{k-j}\left(h_{j}(x), k_{j}(x), g_{j}(x)\right) .
$$

Therefore for all $p \in R_{k}(w) \cap I$ we have $h_{j}(x(p))=k_{j}(x(p))=g_{j}(x(p))=0$, $j=1, \ldots, k$, and $\left(M_{2}^{2}-4 M_{1} M_{3}\right)(x(p), 0) \geq 0$.

Let $\Sigma^{0}(I, w)=\left\{p \in I \mid\right.$ there exists a sequence $\left\{p_{n}\right\}_{n \in \mathbf{N}}$ contained in $(I-\{p\}) \cap$ $R_{k}(w)$ converging to $\left.p\right\}$.

If $p \in \Sigma^{0}(I, w)$ then $h_{j}^{(i)}(x(p))=k_{j}^{(i)}(x(p))=g_{j}^{(i)}(x(p))=0$ for all $i \in \mathbf{N}$ and $j=$ $1, \ldots, k$ and $\left(\partial^{k} / \partial y^{k}\right)(a, b, c)(x(p), 0)=k !\left(M_{1}, M_{2}, M_{3}\right)(x(p), 0) \neq(0,0,0)$. Moreover, we can suppose $M_{i}(x(p), 0) \neq 0$ for $i=1,2,3$ and $\left(M_{2}^{2}-4 M_{1} M_{3}\right)(x(p), 0)>0$ for all $p \in \Sigma^{0}(I, w)$ (Lemma 9.2).

Consider the following set: $\Sigma^{00}(I, w)=\left\{p \in \Sigma^{0}(I, w) \mid\right.$ there exists a sequence $\left\{p_{n}\right\}_{n \in \mathbf{N}}$ contained in $\Sigma^{0}(I, w)-\{p\}$ converging to $\left.p\right\}$. Let us fix $\delta>0$ small enough such that $J \times[-2 \delta, 2 \delta] \subseteq(x, y)(U)$ where $(x, y)(I)=J \times\{0\}$ and $M_{i}(x(p), y) \neq 0$, $i=1,2,3$ and $\left(M_{2}^{2}-4 M_{1} M_{3}\right)(x(p), y)>0$ for all $0<|y| \leq 2 \delta$ and $p \in \Sigma^{00}(I, w)$. 
For each $p \in \Sigma^{00}(I, w)$ consider a closed rectangle $R_{p}=I_{p} \times[-2 \delta, 2 \delta]$ such that $(x(p), 0) \in \operatorname{int}\left(R_{p}\right)$ and

$\max \left\{\left\|h_{i}\right\|_{s},\left\|k_{i}\right\|_{s},\left\|g_{i}\right\|_{s}\right\}<\varepsilon / 2 \lambda_{s}$ on $I_{p}$ for all $i=1, \ldots, k\left(\lambda_{s}\right.$ as in Remark 9.5).

$\left(M_{2}^{2}-4 M_{1} M_{3}\right)(x, y)>0$ for all $(x, y) \in R_{p}$.

$b_{\lambda}^{2}-4 a_{\lambda} c_{\lambda}>0$ in $I_{p} \times([-2 \delta,-\delta] \cup[\delta, 2 \delta])$ for $0 \leq \delta \leq 1$ where $\left(a_{\lambda}, b_{\lambda}, c_{\lambda}\right)=$ $y^{k}\left(M_{1}, M_{2}, M_{3}\right)(x, y)+\lambda(H, K, G)(x, y)$.

Since $\Sigma^{00}(I, w)$ is a compact set, there exist $p_{1}, \ldots, p_{k} \in \Sigma^{00}(I, w)$ with $x\left(p_{1}\right)<$ $\cdots<x\left(p_{k}\right)$ such that $\Sigma^{00}(I, w) \subset \bigcup_{i=1}^{k} \operatorname{int}\left(R_{p_{i}}\right)$ and $R_{p_{i}} \cap R_{p_{i+1}}$ is empty. For $i=$ $1, \ldots, k$ consider two points: $q_{i}^{1}, q_{i}^{2}$ of $\Sigma^{0}(I, w) \cap R_{p_{i}}$ such that $(x, y)\left(\Sigma^{0}(I, w)\right) \cap$ $R_{p_{i}} \subset\left[x\left(q_{i}^{1}\right), x\left(q_{i}^{2}\right)\right] \times\{0\}$.

Let $\tilde{J}=\bigcup_{i=1}^{k}\left[x\left(q_{i}^{1}\right), x\left(q_{i}^{2}\right)\right]$ and $\bar{H}, \bar{K}, \bar{G}$ be the smooth functions defined by

$$
(\bar{H}, \bar{K}, \bar{G})(x, y)=\left\{\begin{array}{l}
(0,0,0) \quad \text { if } x \in \tilde{J} \\
(H, K, G)(x, y) \quad \text { if } x \notin \tilde{J} .
\end{array}\right.
$$

Let $\phi: \mathbf{R} \rightarrow[0,1]$ be a smooth function such that $\phi^{-1}(1)=[-\delta, \delta], \phi^{-1}(0)=$ $\mathbf{R}-]-2 \delta, 2 \delta\left[\right.$, and $\left|\phi^{(i)}(y)\right| \leq 2^{i(i+1) / 2} \cdot \delta^{-1}$ for all $i=1, \ldots, s$ (Lemma 9.3).

Define

$$
\begin{aligned}
(\tilde{a}, \tilde{b}, \tilde{c})(x, y)= & y^{k} \cdot\left(M_{1}, M_{2}, M_{3}\right)(x, y)+\phi(y) \cdot(\bar{H}, \bar{K}, \bar{G})(x) \\
& +(1-\phi(y))(H, K, G)(x) .
\end{aligned}
$$

Then $(\tilde{a}, \tilde{b}, \tilde{c}) \in A_{\infty}((x, y)(U)) ;(\tilde{a}, \tilde{b}, \tilde{c})(x, y)=y^{k} \cdot\left(M_{1}, M_{2}, M_{3}\right)(x, y)$ for all $(x, y) \in \tilde{J} \times[-\delta, \delta] ;(\tilde{a}, \tilde{b}, \tilde{c})=(a, b, c)$ outside $\bigcup_{i=1}^{k} \operatorname{int}\left(R_{p_{i}}\right)$ and $\max \left\{\|\tilde{a}-a\|_{s}\right.$, $\left.\|\tilde{b}-b\|_{s},\|\tilde{c}-c\|_{s}\right\}<\varepsilon$. So, we can get $w_{1} \in \mathscr{F}_{\infty}(M)$, arbitrarily $C^{s}$-close to $w$ such that $\mathscr{M}_{k+1}\left(w_{1}\right) \subset M-\bar{V}$ and $R_{k}\left(w_{1}\right) \cap V$ is a union of a finite number of disjoint compact segments, a finite number of points, and a finite number of sequences of isolated points accumulating on those points. Now the proof follows from previous lemmas.

11. Proof of Proposition 8.3. Let $p$ be an isolated point of $R_{2}(w)$. (respectively, of $\left.R_{1}(w)\right)$ and let $s$ be a positive integer. Let $(x, y):(U, p) \rightarrow\left(\mathbf{R}^{2}, 0\right)$ be a chart with $U \cap R_{2}(w)=\{p\}$ (resp. $U \cap R_{1}(w)=\{p\}$ ) and $(x, y)^{*}(w)=$ $a(x, y) d y^{2}+b(x, y) d x d y+c(x, y) d x^{2}$. It is sufficient to prove that there exists a triple $(\tilde{a}, \tilde{b}, \tilde{c}) \in A_{\infty}((x, y)(U))$ arbitrarily $C^{s}$-close to the triple $(a, b, c)$ and that there exists a neighborhood $V_{1}$ of $(0,0)$ contained in $(x, y)(U)$ such that:

(1) $(\tilde{a}, \tilde{b}, \tilde{c})=(a, b, c)$ outside $V_{1}$.

(2) $(\partial / \partial x)(\tilde{a}, \tilde{b}, \tilde{c})(x, y) \neq(0,0,0)$ or $(\partial / \partial y)(\tilde{a}, \tilde{b}, \tilde{c})(x, y) \neq(0,0,0)$ for all $(x, y)$ $\in V_{1} \cap\left(\tilde{b}^{2}-4 \tilde{a} \tilde{c}\right)^{-1}(0)\left(\operatorname{resp} .\left((\partial \tilde{b} / \partial x)^{2}-4 \partial \tilde{a} / \partial x \cdot \partial \tilde{c} / \partial x\right)(x, y)>0\right.$ and

$$
\begin{aligned}
\left(\left(\left(\frac{\partial \tilde{b}}{\partial x}\right)^{2}-4 \frac{\partial \tilde{a}}{\partial x} \cdot \frac{\partial \tilde{c}}{\partial y}\right)\right. & \left(\left(\frac{\partial \tilde{b}}{\partial y}\right)^{2}-4 \frac{\partial \tilde{a}}{\partial y} \cdot \frac{\partial \tilde{c}}{\partial y}\right) \\
& \left.-\left(\frac{\partial \tilde{b}}{\partial x} \cdot \frac{\partial \tilde{b}}{\partial y}-2 \frac{\partial \tilde{a}}{\partial x} \cdot \frac{\partial \tilde{c}}{\partial y}-2 \frac{\partial \tilde{a}}{\partial y} \cdot \frac{\partial \tilde{c}}{\partial x}\right)^{2}\right)(x, y)>0
\end{aligned}
$$

for all $\left.(x, y) \in V_{1} \cap\left(\tilde{b}^{2}-4 \tilde{a} \tilde{c}\right)^{-1}(0)\right)$. 
11.1. The point $p$ is an isolated point of $R_{1}(w)$. Since $R_{0}(w)$ is contained in $R_{1}(w)$ we have $U \cap \operatorname{Sing}(w)=\{p\}$. First we consider the cases when the vectors $\left(a_{1}, b_{1}, c_{1}\right)=(\partial / \partial x)(a, b, c)(0,0)$ and $\left(a_{2}, b_{2}, c_{2}\right)=(\partial / \partial y)(a, b, c)(0,0)$ are linearly independent. In this case we can suppose that $b_{1}^{2}-4 a_{1} c_{1}>0$ and that $b_{2}^{2}-4 a_{2} c_{2}>0$ (Lemma 9.2.). If $\left(b^{2}-4 a_{1} c_{1}\right)\left(b_{2}^{2}-4 a_{2} c_{2}\right)-\left(b_{1} b_{2}-2 a_{1} c_{2}-2 a_{2} c_{1}\right)^{2}=0$ we define for $\delta>0(\tilde{a}, \tilde{b}, \tilde{c})=(a, b, c)+\delta \Theta(0, b, 0)$, where $\Theta \in b\left(U_{1}, U_{2}\right)$ with $U_{1}$ an open neighborhood of $(0,0)$ and $\bar{U}_{2} \subseteq(x, y)(U)$. Then $(\tilde{a}, \tilde{b}, \tilde{c})$ is in $A_{\infty}((x, y)(U))$, it is arbitrarily $C^{s}$-close to the triple $(a, b, c)$ for $\delta>0$ small enough, $\left(\tilde{b}^{2}-4 \tilde{a} \tilde{c}\right)^{-1}(0) \cap U_{2}=\{(0,0)\}$, and if $(\partial / \partial x)(\tilde{a}, \tilde{b}, \tilde{c})(0,0)=\left(\tilde{a}_{1}, \tilde{b}_{1}, \tilde{c}_{1}\right)$ and $(\partial / \partial y)(\tilde{a}, \tilde{b}, \tilde{c})=\left(\tilde{a}_{2}, \tilde{b}_{2}, \tilde{c}_{2}\right)$ we have

$$
\begin{aligned}
& \left(\tilde{b}_{1}^{2}-4 \tilde{a}_{1} \tilde{c}_{1}\right)\left(\tilde{b}_{2}^{2}-4 \tilde{a}_{2} \tilde{c}_{2}\right)-\left(\tilde{b}_{1} \tilde{b}_{2}-2 \tilde{a}_{1} \tilde{c}_{2}-2 \tilde{a}_{2} \tilde{c}_{1}\right)^{2} \\
& =4\left[(1+\delta)^{2}\left(a_{1} b_{2}-a_{2} b_{1}\right)\left(b_{1} c_{2}-b_{2} c_{1}\right)-\left(a_{1} c_{2}-a_{2} c_{1}\right)^{2}\right] \\
& =4 \delta(2+\delta)\left(a_{1} c_{2}-a_{2} c_{1}\right)^{2}>0 .
\end{aligned}
$$

Now we consider the case when the vectors

$$
(\partial / \partial x)(a, b, c)(0,0) \text { and }(\partial / \partial y)(a, b, c)(0,0)
$$

are not linearly independent. In this case we can suppose that

$$
(\partial a / \partial y)(0,0) \cdot(\partial b / \partial y)(0,0)) \cdot(\partial c / \partial y)(0,0) \neq 0 \quad(\text { Lemma 9.1) }
$$

It follows from the $C^{\infty}$-Weierstrass Preparation Theorem $[\mathbf{1 1}$, p. 152] that there exist an open neighborhood $V_{1}$ of $(0,0)$ contained in $(x, y)(U)$ and smooth functions $h, k, g, M_{1}, M_{2}, M_{3}$ define in $V_{1}$ such that

$$
\begin{aligned}
& a(x, y)=(y-h(x)) M_{1}(x, y), \\
& b(x, y)=(y-k(x)) \cdot M_{2}(x, y), \\
& c(x, y)=(y-g(x)) M_{3}(x, y) \text { with } M_{i}(x, y) \neq 0 \text { for all }(x, y) \in V_{1} \text { and } i=1,2,3 .
\end{aligned}
$$

Let us consider the rectangles

$$
R_{1}=\{(x, y)|| x|\leq r,| y \mid \leq r\}, \quad R_{2}=\{(x, y)|| x|\leq 2 r,| y \mid \leq 2 r\}
$$

with $r>0$ small enough such that $R_{2} \subset V_{1}$. Let $\phi \in b\left(\operatorname{int}\left(R_{1}\right), \operatorname{int}\left(R_{2}\right)\right)$.

Let us consider the cases $M_{1}(0,0) \cdot M_{3}(0,0)>0$ and $M_{1}(0,0) \cdot M_{3}(0,0)<0$ separately.

Case A: $M_{1}(0,0) \cdot M_{3}(0,0)>0$.

Since $b^{2}-4 a c \geq 0$ and $\left(b^{2}-4 a c\right)^{-1}(0) \cap V_{1}=\{(0,0)\}$ we have that for $0<|x| \leq$ $2 r, h(x) \neq g(x)$ and $k(x)$ belong to the open interval whose endpoints are $h(x)$ and $g(x)$. Essentially we only have two cases:

$\left(\mathrm{A}_{1}\right) g(x)<k(x)<h(x)$ for all $x \in[-2 r, 2 r]-\{0\}$,

$\left(\mathrm{A}_{2}\right) h(x)<k(x)<g(x)$ for all $x \in[-2 r, 0[$ and $g(x)<k(x)<h(x)$ for all $x \in] 0,2 r[$.

For Case $\mathrm{A}_{1}$, let $(\tilde{a}, \tilde{b}, \tilde{c})(x, y)=(a, b, c)(x, y)+\delta \phi(x, y)\left(-M_{1}, 0, M_{3}\right)(x, y)$ with $\delta>0$. Then

$$
\begin{aligned}
\left(\tilde{b}^{2}-4 \tilde{a c}\right)(x, y)= & \left(b^{2}-4 a c\right)(x, y) \\
& +4 \delta \phi(x, y)[h(x)-g(x)+\delta \phi(x, y)] M_{1}(x, y) M_{3}(x, y)>0
\end{aligned}
$$

For Case $\mathrm{A}_{2}$, let $(\tilde{a}, \tilde{b}, \tilde{c})(x, y)=(a, b, c)+\delta x \phi(x, y)\left(-M_{1}, 0, M_{3}\right)(x, y)$ with $0<$ $\delta \neq k^{\prime}(0)-h^{\prime}(0)$. Then

$$
\begin{aligned}
\left(\tilde{b}^{2}-4 \tilde{a} \tilde{c}\right)(x, y)= & \left(b^{2}-4 a c\right)(x, y) \\
& +4 \delta x \phi(x, y)[h(x)-g(x)+\delta x \phi(x, y)] \cdot M_{1}(x, y) \cdot M_{3}(x, y) \geq 0
\end{aligned}
$$




$$
\left(\tilde{b}^{2}-4 \tilde{a} \tilde{c}\right)^{-1}(0)=(0,0)
$$

and the vectors $(\partial / \partial x)(\tilde{a}, \tilde{b}, \tilde{c})(0,0),(\partial / \partial y)(\tilde{a}, \tilde{b}, \tilde{c})(0,0)$ are linearly independent (since $\left.\delta \neq k^{\prime}(0)-h^{\prime}(0)\right)$.

Case B: $M_{1}(0,0) \cdot M_{3}(0,0)<0$.

Then for $0<|x| \leq 2 r, k(x)$ is outside the closed interval whose endpoints are $h(x)$ and $g(x)$. Also, essentially we only have two cases:

$\left(\mathrm{B}_{1}\right) k(x)<\min \{g(x), h(x)\}$ for all $x \in[-2 r, 2 r]-\{0\}$,

$\left(\mathrm{B}_{2}\right) k(x)<\min \{g(x), h(x)\}$ for all $x \in[-2 r, 0[$ and $k(x)>\max \{g(x), h(x)\}$ for all $x \in] 0,2 r]$.

For Case $\mathrm{B}_{1}$ let $(\tilde{a}, \tilde{b}, \tilde{c})(x, y)=(a, b, c)(x, y)+\delta \phi(x, y)\left(0, M_{2}, 0\right)(x, y)$ with $\delta>0$. Then

$$
\begin{aligned}
\left(\tilde{b}^{2}-4 \tilde{a} \tilde{c}\right)(x, y)= & \left(b^{2}-4 a c\right)(x, y) \\
& +\delta \phi(x, y)[2(y-k(x))+\delta \phi(x, y)] M_{2}^{2}(x, y)>0
\end{aligned}
$$

since $\tilde{a}(x, y) \cdot \tilde{c}(x, y)>0$ implies $y-k(x)>0$.

For Case $\mathrm{B}_{2}$ let $(\tilde{a}, \tilde{b}, \tilde{c})(x, y)=(a, b, c)(x, y)+\delta x \phi(x, y)\left(0,-M_{2}, 0\right)(x, y)$ with $0<\delta \neq h^{\prime}(0)-k^{\prime}(0)$. Then,

$$
\begin{aligned}
\left(\tilde{b}^{2}-4 \tilde{a} \tilde{c}\right)(x, y)= & \left(b^{2}-4 a c\right)(x, y)-2 \delta x \phi(x, y)(y-k(x)) M_{2}^{2}(x, y) \\
& +\delta^{2} x^{2} \phi^{2}(x, y) M_{2}^{2}(x, y) \geq 0,
\end{aligned}
$$

$\left(\tilde{b}^{2}-4 \tilde{a} \tilde{c}\right)^{-1}(0)=\{(0,0)\}$ and $(\partial / \partial x)(\tilde{a}, \tilde{b}, \tilde{c})(0,0),(\partial / \partial y)(\tilde{a}, \tilde{b}, \tilde{c})(0,0)$ are linearly independent vectors.

11.2. The point $p$ is an isolated point of $R_{2}(w)$. As in 11.1 in this case we can suppose that $a(x, y)=(B(x, y)+x A(x, y)) M_{1}(x, y), b(x, y)=B(x, y) M_{2}(x, y)$, and $c(x, y)=(B(x, y)+x C(x, y)) M_{3}(x, y)$ for all $(x, y)$ on a small open neighborhood $V_{1}$ of $(0,0)$, where $B(x, y)=y^{2}+x y b_{1}(x)+x^{2} b_{2}(x), A(x, y)=y a_{1}(x)+x a_{2}(x)$, and $C(x, y)=y c_{1}(x)+x c_{2}(x)$ with $a_{1}, a_{2}, b_{1}, b_{2}, c_{1}, c_{2}, M_{1}, M_{2}, M_{3}$ smooth functions such that $M_{1}(x, y) \cdot M_{2}(x, y) \cdot M_{3}(x, y) \neq 0$ and $\left(M_{2}^{2}-4 M_{1} M_{3}\right)(x, y)>0$ for all $(x, y) \in V_{1}$.

Case A. The functions $a_{1}, a_{2}, c_{1}, c_{2}$ are flat in 0 . That is, $a_{1}^{(n)}(0)=a_{2}^{(n)}(0)=$ $c_{1}^{(n)}(0)=c_{2}^{(n)}(0)=0$ for all $n \in \mathbf{N}$.

In this case, as in the proof of Lemma 10.2 we find a triple $(\bar{a}, \bar{b}, \bar{c})$ in

$$
A_{\infty}((x, y)(U))
$$

arbitrarily $C^{s}$-close to the triple $(a, b, c)$ such that $(\bar{a}, \bar{b}, \bar{c})=(a, b, c)$ outside a small neighborhood $V_{1}$ of $(0,0)$ and $(\bar{a}, \bar{b}, \bar{c})(x, y)=B(x, y)\left(M_{1}, M_{2}, M_{3}\right)(x, y)$ for all $(x, y) \in V_{1}$. Then if $M_{1}(0,0) \cdot M_{3}(0,0)$ is positive we define $(\tilde{a}, \tilde{b}, \tilde{c})=(\bar{a}, \bar{b}, \bar{c})+$ $\delta \Theta\left(M_{1}, 0,-M_{3}\right)$ and if $M_{1}(0,0) \cdot M_{3}(0,0)$ is negative we define $(\tilde{a}, \tilde{b}, \tilde{c})=(\bar{a}, \bar{b}, \bar{c})+$ $\delta^{\prime} \Theta\left(0, M_{2}, 0\right)$ with $\delta \neq 0$ small and $\Theta \in b\left(U_{1}, V_{1}\right)$, where $U_{1}$ is an open neighborhood of $(0,0)$ with $\bar{U}_{1} \subset V_{1}$.

Case B. There exists $k \in \mathbf{N}$ such that $A(x, y)=x^{k}\left(\alpha_{1}(x) y+\alpha_{2}(x) x\right)$ and $C(x, y)=x^{k}\left(\beta_{1}(x) y+\beta_{2}(x) x\right)$ with $\left(\alpha_{1}, \alpha_{2}, \beta_{1}, \beta_{2}\right)(0) \neq(0,0,0,0)$.

In this case, we will prove that, choosing $\delta \neq 0$ with appropriate sign, the triple $(\bar{a}, \bar{b}, \bar{c})=(a, b, c)+\delta \Theta\left(M_{1}, M_{2}, M_{3}\right)$ is such that $\bar{b}^{2}-4 \bar{a} \bar{c} \geq 0$, where $\Theta$ is a function in $b\left(U_{1}, U_{2}\right)$, with $U_{1}$ and $U_{2}$ small neighborhoods of $(0,0)$. 
For a triple as above, we have

$$
\bar{b}^{2}-4 \bar{a} \bar{c}=b^{2}-4 a c+2 \delta \Theta\left(M_{2} b-2 a M_{3}-2 c M_{1}\right)+\delta^{2} \Theta^{2}\left(M_{2}^{2}-4 M_{1} M_{3}\right) .
$$

Let us consider the functions $h=M_{2} b-2 a M_{3}-2 c M_{1}$ and $\Delta=h^{2}-$ $\left(b^{2}-4 a c\right)\left(M_{2}^{2}-4 M_{1} M_{3}\right)$. Then $h(x, y)=0$ implies $\Delta(x, y) \leq 0 ; h(x, y)=\Delta(x, y)=$ 0 implies $\left(b^{2}-4 a c\right)(x, y)=0$ and $\Delta(x, y)=x^{2(k+1)} \Delta_{1}(x, y) \cdot \Delta_{2}(x, y)$, where

$$
\begin{aligned}
\Delta_{i}(x, y)= & {\left[\lambda(x, y) \alpha_{1}(x)+\left(\gamma(x, y)+(-1)^{i} \mu(x, y)\right) \beta_{1}(x)\right] y } \\
& +\left[\lambda(x, y) \alpha_{2}(x)+\left(\gamma(x, y)+(-1)^{i} \mu(x, y)\right) \beta_{2}(x)\right] x
\end{aligned}
$$

and $\lambda=2 M_{1} M_{3}, \gamma=M_{2}^{2}-2 M_{1} M_{3}, \mu=M_{2} \sqrt{M_{2}^{2}-4 M_{1} M_{3}}$. Therefore $\lambda \cdot \gamma \cdot \mu \neq$ 0 on $V_{1}$, and if $H_{i}=\lambda \alpha_{i}+(\gamma-\mu) \beta_{i}$ and $G_{i}=\lambda \alpha_{i}(\gamma+\mu) \beta_{i}$ we have

(1) $H_{i}(0,0)=G_{i}(0,0)=0$ if and only if $\alpha_{i}(0)=\beta_{i}(0)=0$, and we can suppose

(2) $H_{i}(0,0) G_{i}(0,0)=0$ if and only if $H_{i}(0,0)=G_{i}(0,0)=0$.

(If for example $H_{i}(0,0)=0 \neq G_{i}(0,0)$ defining $(\tilde{a}, \tilde{b}, \tilde{c})=(a,(1+\varepsilon \Theta) b, c)$ with $\varepsilon>0$ small we obtain $\tilde{H}_{i}(0,0) \cdot \tilde{G}_{i}(0,0) \neq 0$.)

So $D \Delta_{1}(0,0) \neq 0$ and $D \Delta_{2}(0,0) \neq 0$ and the curves $\Delta_{1}=0$ and $\Delta_{2}=0$ determine at a small neighborhood $U_{2}$ of $(0,0)$ two sectors where the discriminant $\Delta$ is not negative. Since $\partial^{2} h / \partial y^{2}(0,0) \neq 0$, the function $h$ is always positive or negative where $\Delta$ is positive and we choose $\delta \neq 0$ such that $\delta h(x, y)>0$ for all $(x, y) \in U_{2} \cap \Delta^{-1}(] 0,+\infty[)$.

Observe that if $(\bar{a}, \bar{b}, \bar{c})$ is in $A_{\infty}((x, y)(U))$, taking the neighborhood $U_{1}$ of $(0,0)$ with $\bar{U}_{1} \subset U_{2}$ and $\delta$ small enough we have solved our problem. If $(\bar{a}, \bar{b}, \bar{c})$ is not $A_{\infty}((x, y)(U))$ we define $(\tilde{a}, \tilde{b}, \tilde{c})=(a,(1+\varepsilon \Theta) \bar{b}, \bar{c})$ with $\varepsilon>0$ small enough.

\section{REFERENCES}

1. C. Gutiérrez, Estabilidade estructural para campos de linhas em variedades bi-dimensionais, Doctoral Thesis, IMPA, 1974.

2. C. Gutiérrez and J. Sotomayor, An approximation theorem for immersions with stable configurations of line of principal curvature, Lecture Notes in Math., vol. 1007, Springer, 1983, pp. $332-368$.

3. P. Hartman and A. Wintner, On the singularities in nets of curves defined by differential equations, Amer. J. Math. 75 (1953), 277-297.

4. J. Hubbard and H. Masur, Quadratic differential and foliations, Acta Math. 142 (1979), 221273.

5. A. A. Kadyrov, Critical points of differential equations with unoriented trajectories in the plane, Differential Equations (translated from Differentsial'nye Uravneniya 19 (1983), no. 12, 20392048.

6. J. N. Mather, Stability of $C^{\infty}$-mappings I: The division theorem. Ann. of Math. 87 (1968), 89-104.

7. J. Palis and W. de Melo, Geometric theory of dynamical systems, Springer-Verlag, 1982.

8. M. M. Peixoto, Structural stability on two-dimensional manifolds, Topology 1 (1962), 101-120.

9. H. Rosenberg, Labyrinths in the disc and surfaces, Ann. of Math. 117 (1983), 1-33.

10. Séminaire Orsay, Travaux de Thurston sur les surfaces, Astérisque 66-67 (1979).

11. J. Martinet Singularities of differentiable functions, London Math. Soc. Lecture Notes Series 58, Cambridge Univ. Press, 1982.

12. J. Sotomayor and C. Gutiérrez, Structurally stable configurations of lines of principal curvature, Astérisque 98-99 (1982), 195-215.

Departamento de Matemáticas, facultad de Ciencias, Universidad de Chile, Casilla 653, Santiago, Chile 